\title{
Glutamatergic Innervation onto Striatal Neurons Potentiates GABAergic Synaptic Output
}

\author{
Foteini Paraskevopoulou, Melissa A. Herman, and $@$ Christian Rosenmund \\ Department of Neurophysiology, NeuroCure Cluster of Excellence, Charité Universitätsmedizin, 10117 Berlin, Germany
}

Striatal output pathways are known to play a crucial role in the control of movement. One possible component for shaping the synaptic output of striatal neuron is the glutamatergic input that originates from cortex and thalamus. Although reports focusing on quantifying glutamatergic-induced morphological changes in striatum exist, the role of glutamatergic input in regulating striatal function remains poorly understood. Using primary neurons from newborn mice of either sex in a reduced two-neuron microcircuit culture system, we examined whether glutamatergic input modulates the output of striatal neurons. We found that glutamatergic input enhanced striatal inhibition in vitro. With a glutamatergic partner from either cortex or thalamus, we attributed this potentiation to an increase in the size of quantal IPSC, suggesting a strengthening of the postsynaptic response to GABAergic signaling. Additionally, a differential effect of cortical and thalamic innervation onto striatal GABAergic neurons output was revealed. We observed that cortical, but not thalamic input, enhanced the number of releasable GABAergic synaptic vesicles and morphological synapses. Importantly, these alterations were reverted by blockade of neuronal activity and glutamate receptors, as well as disruption of BDNF-TrkB signaling. Together, our data indicate, for first time, that GABAergic synapse formation in corticostriatal pairs depends on two parallel, but potentially intersecting, signaling pathways that involve glutamate receptor activation in striatal neurons, as well as BDNF signaling. Understanding how cortical and thalamic inputs refine striatal output will pave the way toward dissecting basal ganglia activity in both physiological and pathological conditions.

Key words: BDNF; cell culture; excitatory input; GABAergic neuron; paired recordings; striatum

\section{Significance Statement}

Striatal GABAergic microcircuits are critical for motor function. However, the mechanisms controlling striatal output, particularly at the level of synaptic strength, are unclear. Using two-neuron culture system, we quantified the synaptic output of individual striatal GABAergic neurons paired with a glutamatergic partner and studied the influence of the excitatory connections that are known to be interregionally formed in vivo. We found that glutamatergic input potentiated striatal inhibitory output, potentially involving an increased feedback and/or feedforward inhibition. Moreover, distinct components of glutamatergic innervation, such as firing activity or release of neurotrophic factors were shown to be required for the glutamatergic-induced phenotype. Investigation, therefore, of two-neuron in vitro microcircuits could be a powerful tool to explore synaptic mechanisms or disease pathophysiology.

\section{Introduction}

The striatum is a unique structure containing a high density of GABAergic projection neurons. It serves as the primary gateway

\footnotetext{
Received 0ct. 11, 2018; revised March 24, 2019; accepted March 26, 2019.

Author contributions: F.P., M.A.H., and C.R. designed research; F.P. performed research; F.P. analyzed data; F.P. wrote the paper; M.A.H. and C.R. edited the paper.

F.P. was supported by NeuroCure Cluster of Excellence predoctoral fellowship. This work was supported by Neurocure Excellence Initiative and the German Research Council Deutsche Forschungsgemeinschaft SFB665/B11 to C.R. and HE 7480/2-1 to M.A.H. We thank Bettina Brokowski, Rike Dannenberg, Berit Söhl-Kielczynski, Miriam Petzold, and Katja Pötschke for technical assistance; and members of the C.R. laboratory for discussions.

The authors declare no competing financial interests.

Correspondence should be addressed to Christian Rosenmund at christian.rosenmund@charite.de.

https://doi.org/10.1523/JNEUROSCI.2630-18.2019

Copyright $\odot 2019$ the authors
}

for glutamatergic input to the basal ganglia, and its inhibitory output is associated primarily with motor function (Albin et al., 1989; DeLong, 1990). Based on previous in vivo studies, 95\% of striatal neurons are spiny (medium spiny neurons [MSNs]) and interconnected by local recurrent axon collateral synapses (Czubayko and Plenz, 2002; Tunstall et al., 2002). The MSNs project within basal ganglia networks, such as globus pallidus and substantia nigra, through direct and indirect output pathways (Albin et al., 1989; Gerfen, 1992). In recent years, much attention has been drawn toward unveiling the role of striatal projection neuron output in movement (Cui et al., 2013; Oldenburg and Sabatini, 2015; Rothwell et al., 2015), but despite the advances in our understanding of basal ganglia circuitry, mechanisms con- 
trolling striatal output, particularly at the level of synaptic strength, are still far from clear.

One possible component for shaping the output of striatal neuron synapses is the glutamatergic input onto the neurons themselves. Glutamatergic innervation into striatum mainly originates from cerebral cortex (Kemp and Powell, 1970; McGeorge and Faull, 1989) and thalamus (Groenewegen and Berendse, 1994; Salin and Kachidian, 1998). In particular, motor cortex gives rise to massive excitatory projections that end at the striatum and provide the striatum with information necessary to control motor behavior (Gerfen, 1992; Wilson, 2014). In parallel, thalamic nuclei projections target sensorimotor striatal regions and influence the processing of functionally segregated information (Smith et al., 2004). Previous studies suggest that glutamatergic input not only provides excitation to target GABAergic neurons, but also modulates the size of their inhibitory output, particularly in interneurons through control of synapse formation (Chang et al., 2014). If such modulation is also present at striatal GABAergic neurons, it could have the potential to affect the balance of direct and indirect striatal projections, the strength of lateral inhibition through recurrent connections within striatum, and hence general basal ganglia function.

In the past, efforts have been made to decipher how corticostriatal (CS) and thalamostriatal (TS) projections modulate striatal circuit activity and MSN excitability (Wilson, 1993; Ding et al., 2008). It has been shown in vivo that cortical activity is correlated with MSN transitions from inactive or hyperpolarized to depolarized states, suggesting that prolonged depolarizations are determined by sustained excitatory activity (Stern et al., 1997). Additionally, experiments in acute mouse brain slice revealed that glutamatergic afferents projecting from cortex and thalamus exhibit different short-term synaptic plasticity properties, promoting distinct patterns of MSN spiking (Ding et al., 2008). Although these studies yielded valuable insights, innate technical problems prevent the ability to identify the role of glutamatergic input in regulating striatal activity and to quantify the synaptic output of individual striatal neurons. Dissociated in vitro cell culture systems are at present the most efficient method for recording pairs (Randall et al., 2011) and quantifying the input and output of individual striatal neurons.

In the present study, we used an in vitro dissociated twoneuron interregional microcircuit to explore whether glutamatergic input from cortex or thalamus affects the output of individual striatal GABAergic projection neurons. We recorded connected neurons and evaluated the number of synaptic contacts involved in striatal transmission and identified the synaptic properties of all the possible connections. Furthermore, we explored the contributions of distinct components of glutamatergic innervation, such as introduction of activity or release of BDNF, both of which are crucial for GABAergic synapse formation and function (Hartman et al., 2006; Park and Poo, 2013; Chang et al., 2014). We found that glutamatergic input onto striatal GABAergic neurons did indeed modulate inhibitory synaptic transmission by regulating their output. This process was dependent on action potential generation, glutamatergic synaptic transmission, and BDNF secretion. Together, these results provide insights into basal ganglia physiology and suggest molecular mechanisms through which glutamatergic input modulates striatal output pathways in healthy brain.

\section{Materials and Methods}

Mice and cell culture. Animal housing and use were in compliance with, and approved by, the Animal Welfare Committee of Charité Medical
University and the Berlin State Government Agency for Health and Social Services (License T0220/09). Newborn C57BLJ6/N mice (P0-P2) of both sexes were used for all the experiments.

Primary neurons were seeded and cultured on microisland astrocyte feeder layers that were prepared 2 weeks before the neuronal culture preparation. Astrocytes derived from C57BL/6N mouse cortices ( $\mathrm{P} 0-\mathrm{P} 1)$ were plated on collagen/poly-D-lysine microislands made on agarosecoated coverslips using a custom-built rubber stamp to achieve uniform size (200 $\mu \mathrm{m}$ diameter). For all experiments, neurons from striatum, cortex, or thalamus were digested with papain (Worthington), mechanically dissociated, and plated on astrocytes in a chemically defined medium (Neurobasal-A medium supplemented with Glutamax and B-27; Invitrogen). For two-neuron cultures, neurons were plated at 1:1 ratio and at a total density of $1 \times 10^{4}$ neurons per $35 \mathrm{~mm}$ well. For mass cultures used for $\mathrm{qPCR}$, neurons were plated in absence of astrocytic layer at a density of $5 \times 10^{5}$ to $6 \times 10^{5}$ neurons per $35 \mathrm{~mm}$ well.

For drug treatment experiments, neurons were treated with $0.5 \mu \mathrm{M}$ TTX (Tocris Bioscience); $2 \mu \mathrm{M}$ NBQX (Tocris Bioscience) and $100 \mu \mathrm{M}$ APV (Tocris Bioscience); 200 nм K252a (Tocris Bioscience); BDNF neutralizing antibody $\alpha$-BDNF (1:100; Millipore) and human recombinant BDNF (50 ng/ml; PeproTech) at DIV 3, 7, 11.

Membrane dye labeling. To identify the cell region of origin in electrophysiological recordings, dissociated tissues for the two-neuron cultures were labeled with different fluorescent membrane dyes (PKH26 red or PKH67 green) using a fluorescent cell linker kit for general membrane labeling (Sigma-Aldrich).

Electrophysiology. Paired whole-cell voltage-clamp recordings were performed with a Multiclamp 700B amplifier (Molecular Devices) under the control of Clampex 10.2 (Molecular Devices) between DIV 12 and 15. Data were digitally sampled at $10 \mathrm{kHz}$ and low-pass Bessel filtered at 3 $\mathrm{kHz}$ with an Axon Digidata 1440A digitizer (Molecular Devices). Series resistance was compensated at $70 \%$, and only cells with $<12 \mathrm{~m} \Omega$ resistance were included. All experiments were performed at room temperature $\left(23^{\circ} \mathrm{C}-24^{\circ} \mathrm{C}\right)$.

During recordings, neurons were immersed in standard extracellular solution consisting of the following (in $\mathrm{mM}$ ): $140 \mathrm{NaCl}, 2.4 \mathrm{KCl}, 10$ HEPES, 10 glucose, $4 \mathrm{MgCl}_{2}$, and $2 \mathrm{CaCl}_{2}$. The patch pipette internal solution contained the following (in mM): $136 \mathrm{KCl}, 17.8 \mathrm{HEPES}, 1 \mathrm{EGTA}$, $0.6 \mathrm{MgCl}_{2}, 4$ ATP-Mg, 0.3 GTP-Na, 12 phosphocreatine, and $50 \mathrm{U} / \mathrm{ml}$ phosphocreatine kinase. Both solutions were adjusted to $\mathrm{pH} 7.4$ with osmolarity at $300 \mathrm{mOsm}$. Borosilicate glass patch pipettes were pulled using a multistep puller (P-87, Sutter Instruments) using conditions that kept pipette tip resistance between 2 and $5 \mathrm{M} \Omega$.

Action potential-evoked postsynaptic currents (PSCs) were triggered by a $2 \mathrm{~ms}$ somatic depolarization from $-70 \mathrm{mV}$ (holding potential) to 0 $\mathrm{mV}$. Neurons were stimulated at $0.1 \mathrm{~Hz}$ in standard external solution to measure basal-evoked EPSCs (excitatory postsynaptic currents) or IPSCs (inhibitory postsynaptic currents). The kinetics of the evoked responses and AMPA receptor antagonist ( $3 \mu \mathrm{M}$ NBQX; Tocris Bioscience) were used to verify glutamatergic or GABAergic identities. Spontaneous release of GABAergic cells only was determined by detecting mIPSCs (miniature inhibitory postsynaptic currents) in the presence of NBQX, for $20-40 \mathrm{~s}$ at $-70 \mathrm{mV}$ with the help of a template-based algorithm in Axograph X version 1.6.4 (Axograph Scientific). Data were filtered at 1 $\mathrm{kHz}$, and the threshold for detection was set at 3 times the baseline SD from a template of $0.5 \mathrm{~ms}$ rise and $18 \mathrm{~ms}$ decay time for GABAergic events. Membrane capacitance measurements were obtained from the membrane test function in pClamp (Molecular Devices). Readily releasable pool (RRP) size of striatal cells (GABAergic only) was assessed by measuring the charge transfer of the transient synaptic current induced by a $5 \mathrm{~s}$ application of $500 \mathrm{~mm}$ hypertonic sucrose in standard extracellular solution supplemented with NBQX (Rosenmund et al. 1996). The output RRP was the sum of the autaptic and heterosynaptic RRPs in mixed pairs (Glu-GABA). In the case of control striatal pairs (GABAGABA), a configuration in which the contribution of each neuron's output RRP is not pharmacologically distinguishable, the output RRP was divided by half for each GABAergic neuron, assuming that both RRPs are of equal size. The number of synaptic vesicles in the RRP of neurons was calculated by dividing the sucrose charge by the charge of the average 
miniature event of the same neuron. Similarly, the release probability of a single synapse (Pvr) was calculated as the ratio of input-evoked response charge (autaptic and heterosynaptic connections ending at each postsynaptic neuron) to output RRP charge of the same neuron. Shortterm plasticity was examined by evoking either 50 synaptic responses at 5 $\mathrm{Hz}$ or 2 responses at $20 \mathrm{~Hz}$ (interstimulus interval of $50 \mathrm{~ms}$ ) to calculate a paired-pulse ratio (PPR; response $2 /$ response 1 ). Data were analyzed in Axograph X (Axograph Scientific), Excel (Microsoft), and Prism (GraphPad). All experiments were performed blinded to the experimental groups, and coverslips were always randomized to drug treatments in all experiments. Cells were excluded for further analysis when neither sponanteous release nor hypertonic sucrose-evoked release was detected.

Immunocytochemistry. At DIV 12-15 (unless otherwise noted), neurons were rinsed with PBS and fixed in $4 \% \mathrm{w} / \mathrm{v}$ PFA in PBS, pH 7.4 for 10 min in room temperature, after which they were washed 3 times in PBS. Following permeabilization and blocking with $5 \% \mathrm{v} / \mathrm{v}$ normal donkey serum in PBS with $0.1 \%$ Tween (PBST) for $1 \mathrm{~h}$, cells were incubated with primary antibodies of interest overnight at $4^{\circ} \mathrm{C}$. We used the following antibody dilutions: (1) mouse anti-vesicular GABA transporter (VGAT) (1:1000; Synaptic Systems), (2) chicken anti-microtubule-associated protein 2 (MAP2) (1:2000; Millipore), (3) guinea pig anti-vesicular glutamate transporter 1(VGLUT1) (1:4000; Synaptic Systems), and (4) guinea pig anti-vesicular glutamate transporter 2(VGLUT2) (1:1000; Synaptic Systems). Coverslips were then washed 3 times with PBST for 15 min each, and primary antibodies were labeled with secondary AlexaFluor-488, -555, or -647 (1:500; Jackson ImmunoResearch Laboratories) antibodies for $1 \mathrm{~h}$ at room temperature. Finally, coverslips were washed twice with PBST and twice with PBS for $15 \mathrm{~min}$, each after that they were mounted on glass slides with Mowiol.

Quantification of neuronal morphology. For morphological analysis, 16-bit images were acquired on an Olympus IX81 inverted epifluorescence microscope at $20 \times$ optical magnification with a CCD camera (Princeton MicroMax; Roper Scientific) and MetaMorph software (Molecular Devices). At least three independent cultures were imaged and analyzed blindly to groups for every experiment. All images were acquired using equal exposure times and subjected to uniform background subtraction (radius of 30 pixels) and optimal threshold adjustment.

To determine total dendrite length, MAP2-positive processes were traced with the NeuronJ plugin (Meijering et al., 2004) and crosssectional area across the MAP2-positive cell body was measured to estimate the area of neuronal somata. For synapse number quantification, GABAergic synapses were identified by immunoreactivity to VGAT antibody, while glutamatergic synapses in coriticostraital pairs were identified by immunoreactivity to VGLUT1 antibody and glutamatergic synapses in TS pairs identified by immunoreactivity to VGLUT2 antibody according to the reported vesicular glutamate transporter isoform expression pattern (Fremeau et al., 2001; Fujiyama et al., 2001). The total number of GABAergic and glutamatergic synapses was quantified by manually counting the VGAT and VGLUT1 (cortex) or VGLUT2 (thalamus) fluorescent punta, respectively. After background subtraction with a rolling ball of a radius of 30 pixels and threshold adjustment, images were converted to binary using ImageJ plugin. Only puncta with $<6$ pixels $\wedge 2$ were included in the analysis. For heterotypic pairs, the total number of VGAT puncta represented all the synapses of the GABAergic partner, while in homotypic striatal pairs the same measure represented the synapses from both GABAergic cells. Therefore, the total number of VGAT puncta was divided by half, in the case of striatal pairs. Raw values were exported to Prism (GraphPad) for further analyses.

$q R T-P C R$. Total cellular RNA was extracted using QIAzol Lysis Reagent (QIAGEN) reagent and followed by cDNA synthesis using M-MLV reverse transcriptase (Promega) and random hexanucleotides. The mRNA expression levels of each sample were normalized to tubulin (Tubb3) mRNA content. For detection of the amplification products in Tubb3 and Bdnf RT-PCR, we used SYBR Green dye-based PCR amplification (Thermo Fisher Scientific) and the QuantStudio 3 detection system (Applied Biosystems). The following sequence-specific primers (MWG Biotech) were used: Tubb3 forward, 5'-GCGCATCAGCGTAT ACTACAA-3' and reverse, 5'-CATGGTTCCAGGTTCCAAGT-3';
Bdnf forward, 5'-GACGACATCACTGGCTGACA-3' and reverse, 5'-CAAGTCCGCGTCCTTATGGT-3'.

Statistical analysis. Power analysis was performed using the pwr $R$ package (https://github.com/heliosdrm/pwr) before experimental design to estimate the sample size for detecting differences, if exist, between conditions. A statistical power of 0.8 was set for pairwise comparisons, with a two-sided Type I error rate of 0.05 and a medium effect size of 0.5 (Cohen, 1988). To evaluate our methodological approach, at the end of the experiment, the statistical power was recalculated ( $\geq 0.9$ in all cases) using the empirical mean values of the compared groups, the SD, the $\alpha$, and sample size. To test the normality of the data, we used the D'Agostino-Pearson test. Student's $t$ test for independent groups and one-way ANOVA using Tukey HSD post hoc test were used to assign the level of statistical significance between conditions. When parametric assumptions were violated, we performed Mann-Whitney $U$ test and Kruskal-Wallis test coupled with Dunn's post hoc test. All analyses were performed in Prism version 7 (GraphPad). Data are presented as mean \pm SEM.

\section{Results}

To investigate the interaction between excitatory (cortical or thalamic) and striatal inhibitory neurons on the cellular level, we used two-neuron microisland cultures. In each condition, we systematically altered the neuron type (glutamatergic or GABAergic) and the tissue origin (cortex/thalamus or striatum) of each neuron. The tissue origin of each cell was determined by membrane dye-labeling of dissociated neurons before plating (see Materials and Methods), and neurotransmitter type was determined by kinetics of the postsynaptic responses (Fig. 1). This in vitro approach allowed us for an unambiguous and quantitative electrophysiological (455 pairs) and morphological (191 pairs) characterization of synaptic connectivity and function of neurons of known identity.

\section{Glutamatergic innervation onto striatal neurons increases GABAergic synaptic output}

Synapses originating from cortical or thalamic neurons onto MSNs provide most of the excitatory glutamatergic input onto the striatum (Smith and Bolam, 1990; Doig et al., 2010). Hence, we decided to test for changes in synaptic connectivity and strength with the innervation of striatal GABAergic neurons with either of the two inputs. To do so, we used a two-neuron microcircuit culture system and compared control striatal (SS) (only GABAergic; homotypic) pairs to CS or TS (glu-GABA; heterotypic) pairs (Fig. 1; Fig. 1-1, available at https://doi.org/10.1523/ JNEUROSCI.2630-18.2019.f1-1). Because in this configuration, each neuron forms a synaptic connection with its partner (heterosynapses), as well as with itself (autapses), four distinct synaptic connections are present in each pair (Fig. 1A$C)$. To demonstrate that all changes in GABAergic output are specific to the presence of glutamatergic partner, we used single striatal neurons, growing on glial islands, as a second control, and compared their properties with homotypic GABA pairs.

First, we analyzed the impact of glutamatergic innervation on action-potential-evoked IPSCs generated by striatal GABAergic neurons, by looking at the total autaptic and heterosynaptic responses. Both cells in each pair configuration (Fig. $1 A-C$ ) were measured simultaneously using whole-cell patch-clamp of their somata, and subsequent brief depolarizations were applied to elicit an action potential in either of the connected neurons. Striatal neurons paired with either a cortical or thalamic glutamatergic partner revealed an approximately twofold increase in evoked IPSC total amplitude (CS: $-21.14 \pm 3.12 \mathrm{nA}, n=29, p=0.006$; TS: $-19.13 \pm 3.29 \mathrm{nA}, n=25, p=0.026$, Kruskal-Wallis test), compared with those paired with another striatal GABAergic 
A

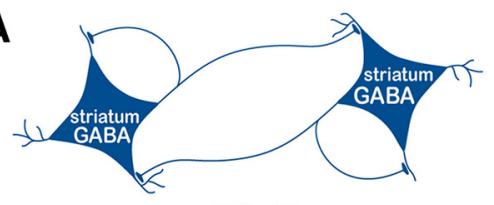

D

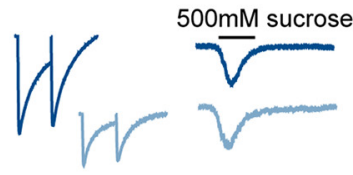

B

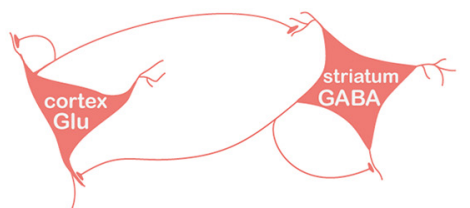

E

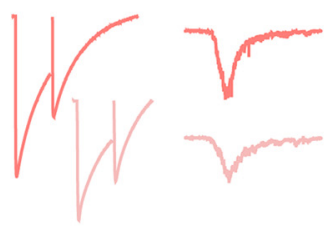

C

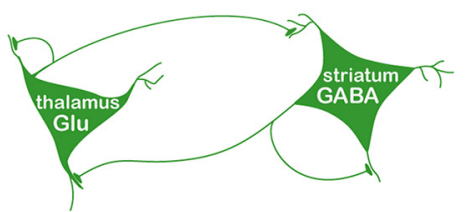

F

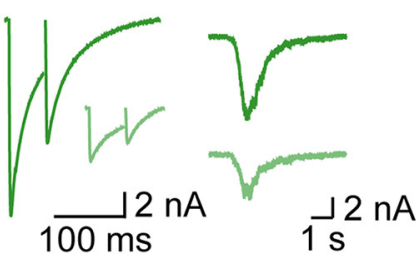

G

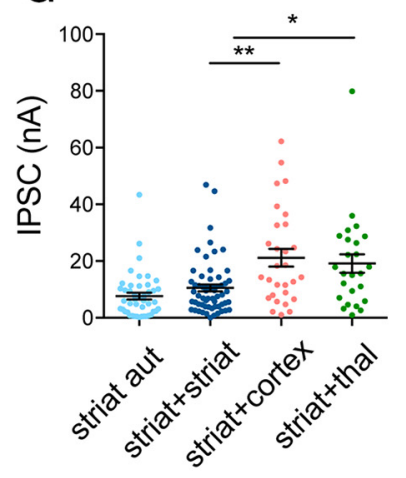

K
H
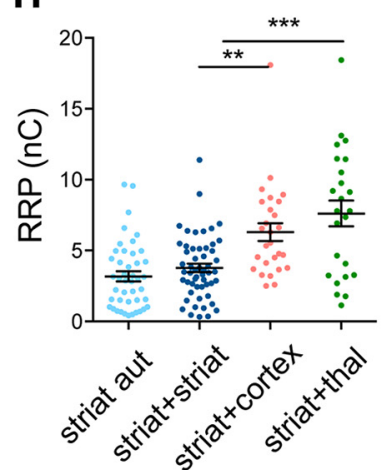

L
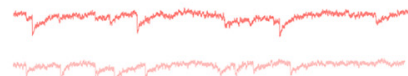

N

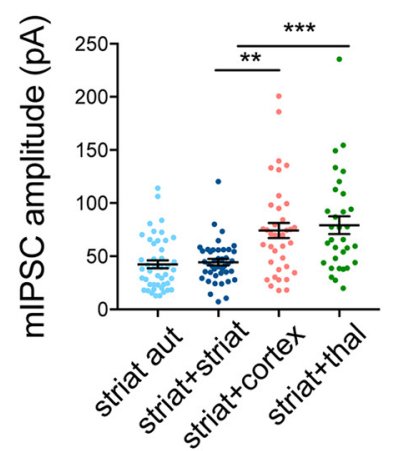

$\mathbf{R}$
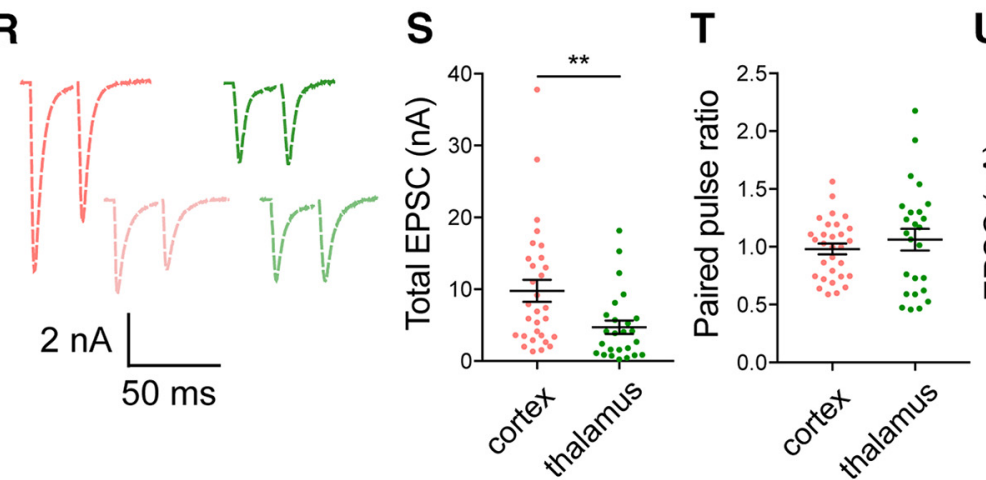

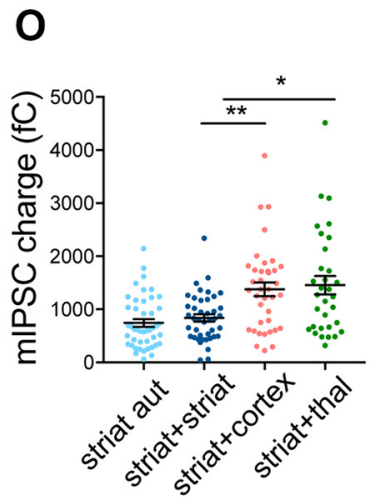

$\mathbf{P}$

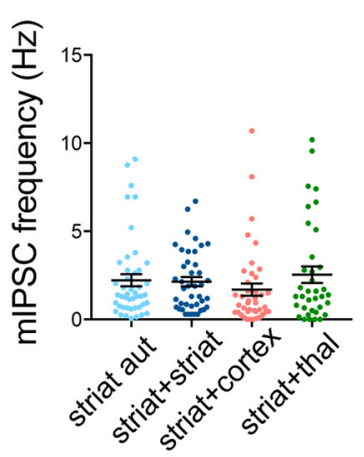

J
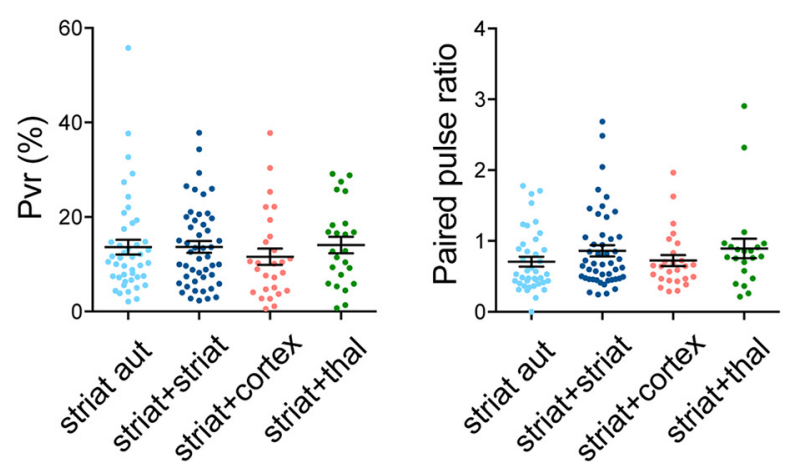

\section{M}

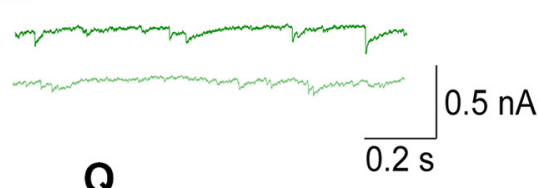

$\mathbf{U}$
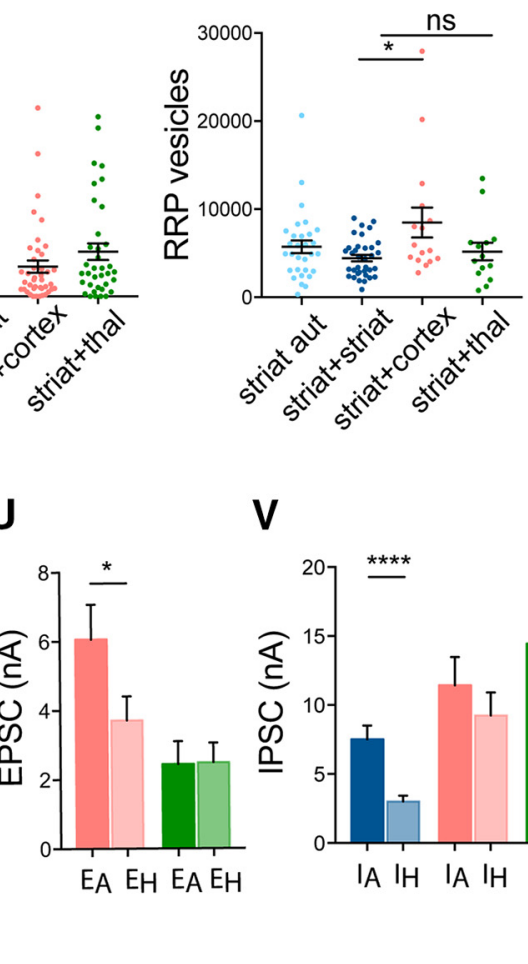

V

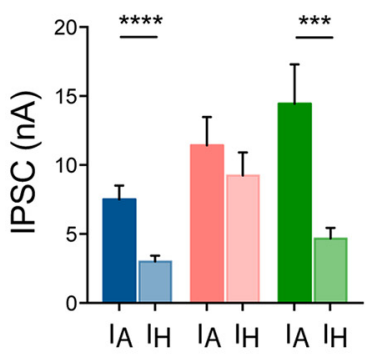

Figure 1. Striatal GABAergic output is modulated by glutamatergic input. A-C, Schematic diagram illustrating autaptic and heterosynaptic connections in striatal (dark blue represents GABAergic only), CS (pink represents glu-GABA), and TS (green represents glu-GABA) pairs. D-Q, Functional analysis of striatal autapses (light blue traces and dots), striatal pairs (blue traces and dark blue dots), CS pairs (pink traces and dots), and TS pairs (green traces and dots). $\boldsymbol{D}-\boldsymbol{F}$, Representative traces of GABAergic response to paired pulse stimulation with $50 \mathrm{~ms}$ interstimulus interval and to a $5 \mathrm{~s}$ pulse of $500 \mathrm{~mm}$ hypertonic sucrose solution (dark represents autaptic; light represents heterosynaptic). G-J, Scatter plots showing total evoked IPSC amplitudes (G), RRP size (Figure legend continues.) 
neuron (SS IPSC: $-10.51 \pm 1.23 \mathrm{nA}, n=60$ ) (Fig. $1 D-G$ ). These findings suggest that glutamatergic input increases the magnitude of GABAergic output of striatal neurons.

The magnitude of the evoked IPSC depends on the number of fusion competent vesicles RRP, the efficiency of the calciumtriggered fusion of synaptic vesicles (vesicular release probability, Pvr) and the postsynaptic response to the release of an individual vesicle (quantal size) (del Castillo and Katz, 1954; Reim et al., 2001). To determine which of these parameters underlie the increase in evoked release of striatal neurons in mixed pairs, first, we looked at readily releasable vesicles in the presence of the glutamate receptor antagonist, NBQX. When we measured RRP size by integrating the charge resulting from the pulsed application of hypertonic sucrose supplemented with NBQX, we found that the total RRP size in striatal neurons connected with glutamatergic neurons was increased by $67 \%$ for CS and $102 \%$ for TS pairs compared with striatal neuron pairs (SS: $-3.77 \pm 0.30 \mathrm{nC}$, $n=54$; CS: $-6.30 \pm 0.62 \mathrm{nC}, n=27, p=0.002$; TS: $-7.61 \pm$ $0.91 \mathrm{nC}, n=24, p=0.001$, Kruskal-Wallis test) (Fig. $1 D-F, H$ ). This suggests that the magnitude of the increase in the RRP was comparable with the increase seen in the IPSC (101\% and 82\%, respectively). We next examined whether glutamatergic input affects the presynaptic release efficiency by calculating the probability of single vesicle to undergo exocytosis (Pvr; IPSC charge divided by sucrose-evoked charge) or the PPR. Our data revealed no changes in Pvr and PPR (SS: $13.66 \pm 1.24 \%, n=48$; CS: $11.64 \pm 1.79 \%, n=27, p=0.625$; TS: $14.07 \pm 1.76 \%, n=24, p>$ 0.999 , Kruskal-Wallis test) (Fig. $1 I, J$ ), implying that glutamatergic input affects RRP size without altering presynaptic calciumdependent release efficiency.

To assess quantal size, we analyzed spontaneous release events, or mIPSCs. We noted that the mean mIPSC amplitude of striatal neurons in mixed pairs was significantly greater than controls by $\sim 70 \%$ (SS: $-44.31 \pm 3.04 \mathrm{pA}, n=42$; CS: $-74.24 \pm 7.07$ $\mathrm{pA}, n=38, p=0.002$; TS: $-79.17 \pm 8.16 \mathrm{pA}, n=32, p=0.001$, Kruskal-Wallis test), as was the mIPSC charge (SS: $-838.10 \pm$ $68.96 \mathrm{fC}, n=42$; CS: $-1379 \pm 129.2 \mathrm{fC}, n=38, p=0.004$; TS: $-1454 \pm 172.5 \mathrm{fC}, n=32, p=0.01$, Kruskal-Wallis test) (Fig. $1 \mathrm{~K}-\mathrm{O})$. To eliminate the possibility that the mIPSC increase in mixed pairs is due to the nature of postsynaptic responses in glutamatergic neurons, we compared the mIPSC amplitudes recorded only in the striatal GABAergic neurons in both homotypic and mixed pairs. We found that the GABAergic mIPSCs recorded in striatal neurons (autaptic) were also significantly increased by $\sim 65 \%$ (SS: $-45.26 \pm 2.9 \mathrm{pA}, n=49$; CS: $-75.32 \pm 6.6 \mathrm{pA}, n=$ 41, $p<0.0001$; TS: $-72.96 \pm 11.44 \mathrm{pA}, n=15, p=0.043$, Kruskal-Wallis test), suggesting that glutamatergic input alters the striatal neuron's sensitivity to GABA itself and thus potentiates the collateral feedback inhibition. The frequency of inhibitory miniature events was not changed despite the presence of

\footnotetext{
(Figure legend continued.) $\quad(\boldsymbol{H})$, Pvr\% (I), and PPR $(\boldsymbol{J}) . \boldsymbol{K}-\boldsymbol{M}$, Representative traces showing miniature postsynaptic current activity (dark represents autaptic; light represents heterosynaptic). $\boldsymbol{N}-\boldsymbol{Q}$, Scatter plots showing mean mIPSC amplitudes $(\boldsymbol{N})$, charge $(\boldsymbol{O})$, frequency $(\boldsymbol{P})$, and RRP vesicle number $(\mathbf{Q}) . \boldsymbol{R}$, Representative traces of glutamatergic response to paired pulse stimulation with $25 \mathrm{~ms}$ interstimulus interval (dark represents autaptic; light represents heterosynaptic; pink represents (S pairs; green represents TS pairs). S, $I$, Scatter plots showing total evoked EPSC amplitudes $(\boldsymbol{S})$ and PPR ( $\boldsymbol{T})$. $\boldsymbol{U}, \boldsymbol{V}$, Bars graphs showing the mean PSC amplitude of autaptic and heterosynaptic responses of glutamatergic $(\boldsymbol{U})$ and GABAergic neurons in homotypic or heterotypic pairs $(\boldsymbol{V})$. Data are mean \pm SEM. ns refers to not significant, ${ }^{*} p \leq 0.05$, ${ }^{* *} p \leq 0.01,{ }^{* * *} p \leq 0.001,{ }^{* * * *} p \leq 0.0001$. See also Figure 1-1 (available at https://doi.org/10.1523/JNEUROSCI.2630-18.2019.f1-1).
}

$\leftarrow$ glutamatergic input (SS: $2.15 \pm 0.26 \mathrm{~Hz}, n=42$; CS: $1.70 \pm 0.35$ $\mathrm{Hz}, n=41, p=0.121$; TS: $2.54 \pm 0.47 \mathrm{~Hz}, n=36, p>0.999$, Kruskal-Wallis test) (Fig. $1 P$ ). We then calculated the total number of vesicles in the RRP by dividing the total output RRP charge by the average charge of the miniature events from each neuron. Our analysis showed that the mean number of synaptic vesicles contained in the RRP of striatal neurons was significantly increased only in the case of CS pairs by $91 \%$, but not in that of TS (SS: $4445 \pm 362, n=35$; CS: $8498 \pm 1700, n=16, p=0.04$; TS: $5197 \pm 990, n=14, p>0.999$, Kruskal-Wallis test) (Fig. 1Q). This suggests a differential effect of cortical and thalamic innervation in striatum, in a way that only cortical input causes an increase in the number of RRP vesicles, whereas the increased RRP size in TS connections reflects the postsynaptic effect as supported by increased mIPSC size.

\section{Different connectivity patterns revealed between CS and TS pairs}

To gain a better insight into the differential effect of cortical and thalamic input onto striatal neurons, we analyzed the strength of synaptic connection in mixed pairs by assessing the total excitatory output of glutamatergic partners (Fig. $1 R-T$ ). We noted that the total EPSC amplitude of cortical neurons was $52 \%$ greater than the thalamic one (CS: $-9.78 \pm 1.53 \mathrm{nA}, n=30$; TS: $-4.72 \pm 0.95 \mathrm{nA}, n=25, p=0.006$, Mann-Whitney test) (Fig. $1 R, S)$. However, when applying the paired pulse stimuli, the response ratios were indistinguishable (CS: $0.98 \pm 0.05, n=30$; TS: $1.06 \pm 0.09 \mathrm{nA}, n=25, p=0.42$, $t$ test) (Fig. $1 T$ ), indicating that the relatively higher output from the cortical neurons onto striatal GABAergic neurons was not due to higher release efficacy, but was likely caused by a higher number of synaptic connections.

Excitation of either neuron within a pair resulted in autaptic (dark color bars) and heterosynaptic (light color bars) evoked responses from each neuron (Fig. $1 U, V$ ). Glutamatergic neurons from cortex, in mixed pairs, evoked on average bigger autaptic versus heterosynaptic responses (CS autaptic: $-6.07 \pm 1.04 \mathrm{nA}$; heterosynaptic: $-3.71 \pm 0.70 \mathrm{nA}, n=30, p=0.04$, MannWhitney test), while thalamic partners showed comparable autaptic and heterosynaptic EPSC amplitudes (TS autaptic: $-2.44 \pm 0.66 \mathrm{nA}$; heterosynaptic: $-2.49 \pm 0.56 \mathrm{nA}, n=25, p=$ 0.984, Mann-Whitney test) (Fig. $1 U$ ). To investigate whether the change in striatal GABAergic output was general or target specific, we compared the autaptic and heterosynaptic connections of striatal neurons across the different groups (Fig. $1 \mathrm{~V}$ ). In accordance with previous findings from hippocampal interneurons (Liu et al., 2009, 2013; Chang et al., 2014), we found that autaptic responses of striatal GABAergic neurons were twofold higher than heterosynaptic in homotypic pairs (SS autaptic: $-7.51 \pm$ $0.99 \mathrm{nA}$; heterosynaptic: $-3.01 \pm 0.41 \mathrm{nA}, n=60, p<0.0001$, Mann-Whitney test) (Fig. $1 \mathrm{~V}$ ). Interestingly, while the total IPSC output (autaptic and heterosynaptic) from striatal neurons was significantly greater in the presence of either cortical or thalamic partner (Fig. 1G), the preference for autaptic connections was only preserved in TS connections (Fig. $1 \mathrm{~V}$ ). On the other hand, upon cortical innervation, striatal neurons showed a specific increase in heterosynaptic response (Fig. $1 V ; p=0.0045$ ). This could imply a target specific modulation of striatal neuron output in the CS microcircuit.

\section{Cortical input increases the number of GABAergic synapses in striatal neurons}

Our electrophysiological experiments indicated a differential effect of cortical and thalamic innervation on striatal neurons func- 


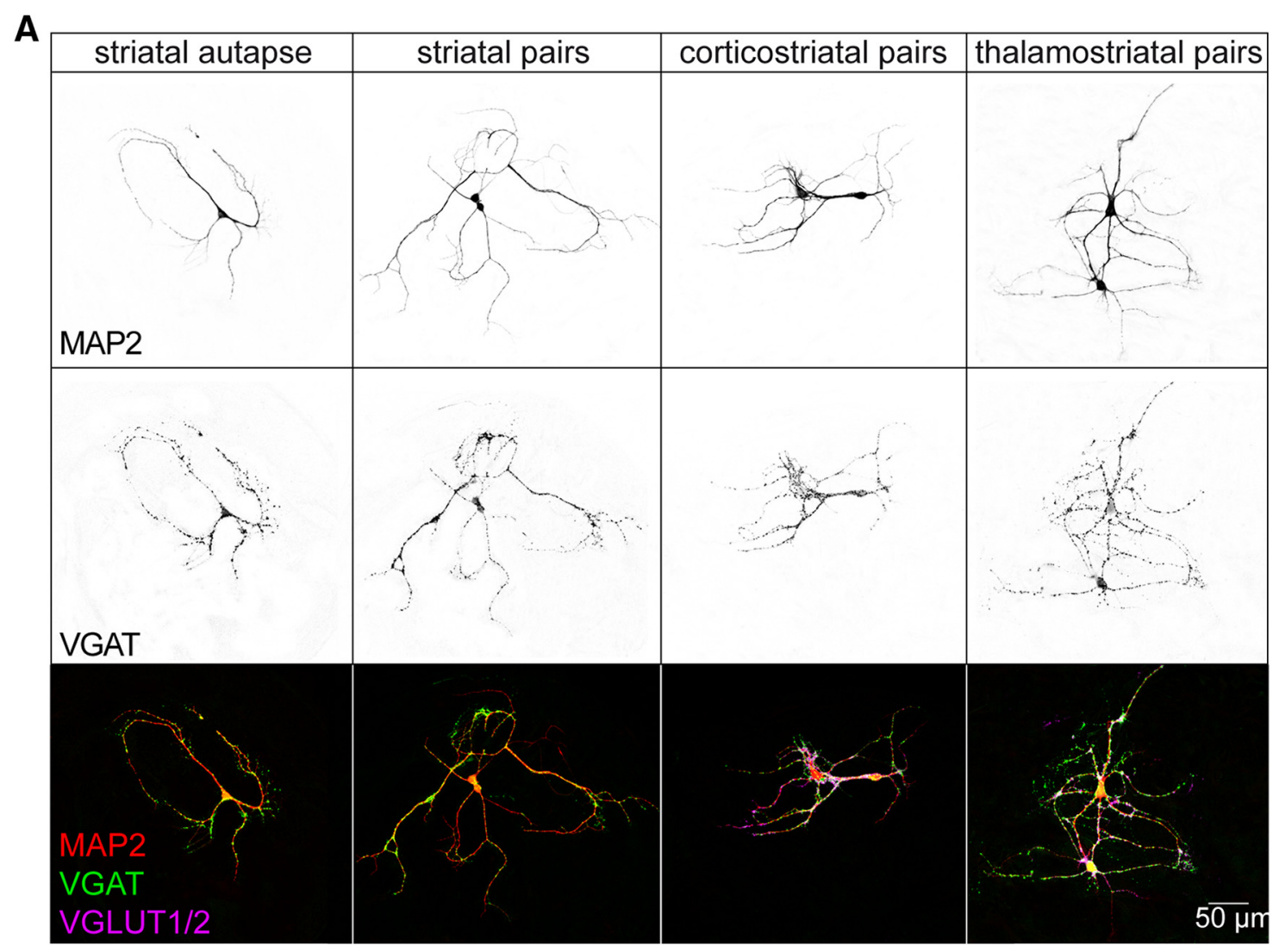

B

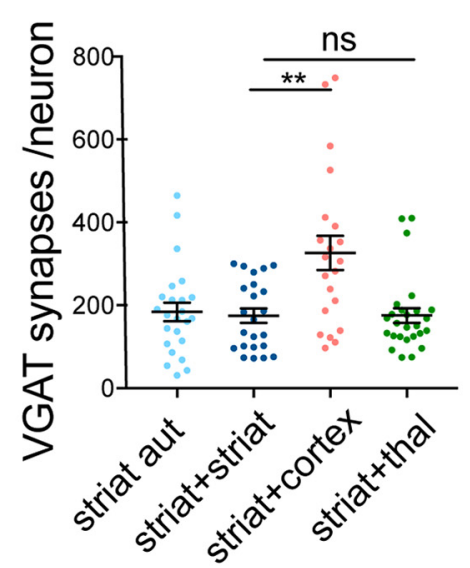

c

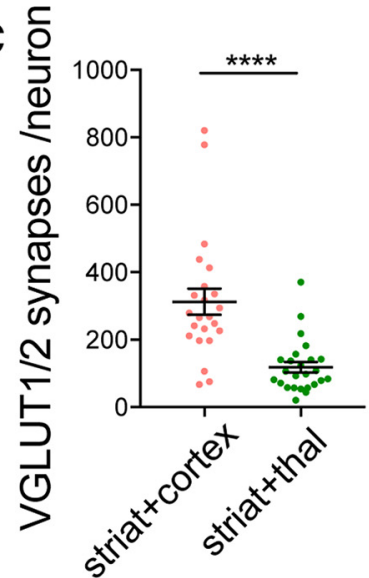

D

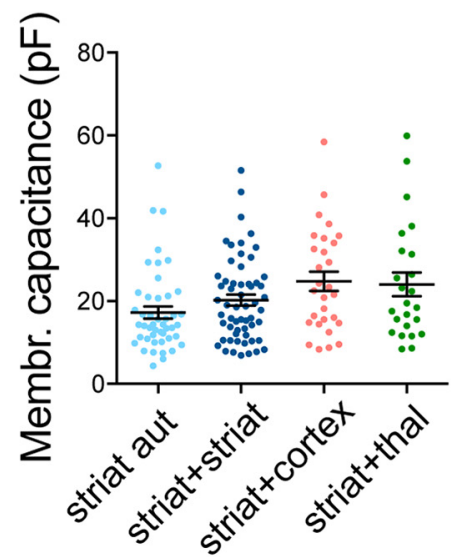

Figure 2. Cortical input increases the number of GABAergic synapses in striatal neurons. $\boldsymbol{A}, \boldsymbol{B}$, Morphological analysis of striatal autapses (light blue dots), striatal pairs (dark blue dots), CS pairs (pink dots), and TS pairs (green dots). $\boldsymbol{A}$, Representative images of neuronal morphology showing immunoreactivity for MAP2, VGAT, and VGLUT1 (cortical synapses) or VGLUT2 (thalamic synapses). $\boldsymbol{B}, \boldsymbol{C}$, Scatter plots showing the number of VGAT synapses per neuron $(\boldsymbol{B})$, the number of VGLUT1 or VGLUT2 synapses per neuron ( $\boldsymbol{C}$, and mean membrane capacitance measurements as obtained from the membrane test $(\boldsymbol{D})$. Data are mean \pm SEM. ns refers to not significant, ${ }^{* *} p \leq 0.01,{ }^{* * *} p \leq 0.0001$. See also Figure $2-1$ (available at https://doi.org/10.1523/ JNEUROSCI.2630-18.2019.f2-1).

tion. While we observed an increase in the magnitude of the RRP output size of striatal GABAergic neurons cocultured with either glutamatergic partner, only striatal neuron paired with a cortical glutamatergic neuron exhibited an increase in the number of RRP vesicles (Fig. $1 H, Q$ ). The increase in RRP vesicles could be a result of either a higher number of vesicles per synapse or by an increase in the number of synapses. To determine the locus of RRP vesicle increase in striatal GABAergic neurons paired with a cortical glutamatergic neuron, we examined the morphology of two-neuron cultures immunolabeled with antibodies against: (1) the predominant subtype of VGLUT in cortical or thalamic neurons, VGLUT1 or VGLUT2, respectively, to mark glutamatergic synapses (Fremeau et al., 2001; Fujiyama et al., 2001); (2) VGAT to mark GABAergic synapses; and (3) MAP2 to visualize dendrites. We quantified the number of VGAT-positive puncta in mixed and control pairs, and found near doubling (187\%) in the number of GABAergic synapses made by striatal neurons in CS pairs, but not in TS pairs (103\%), compared with control neurons (SS: $174.8 \pm 17.48, n=23$; CS: $326.3 \pm 41.52, n=21, p=$ 0.009; TS: $175.4 \pm 17.72, n=26, p>0.999$, Kruskal-Wallis test) (Fig. 2A,B; Fig. 2-1, available at https://doi.org/10.1523/ JNEUROSCI.2630-18.2019.f2-1). This is consistent with our findings from electrophysiological analysis and suggests that the larger number of RRP vesicles of striatal GABAergic neurons in 
A

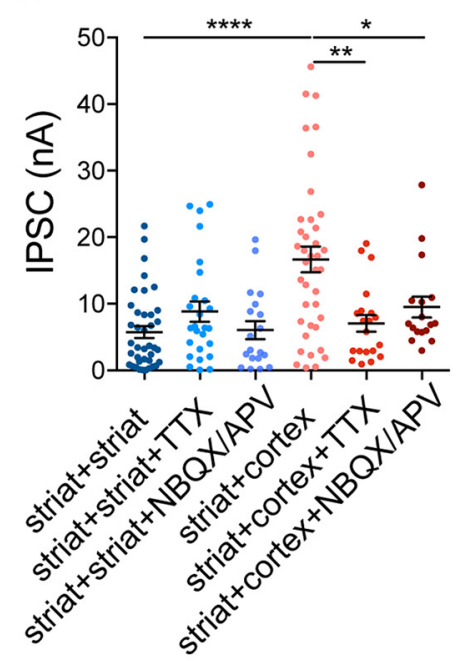

D

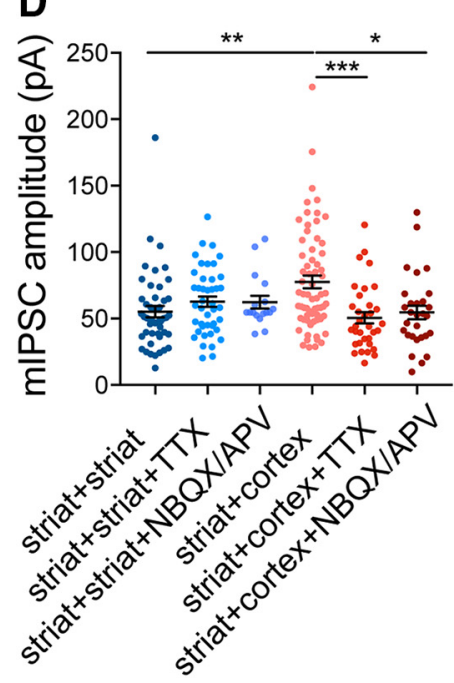

B

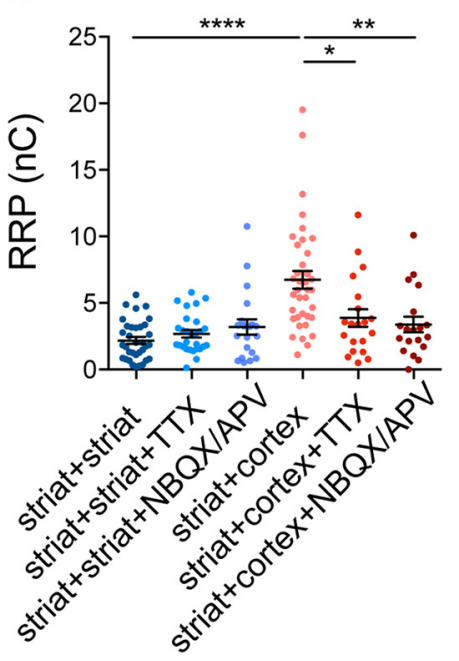

E

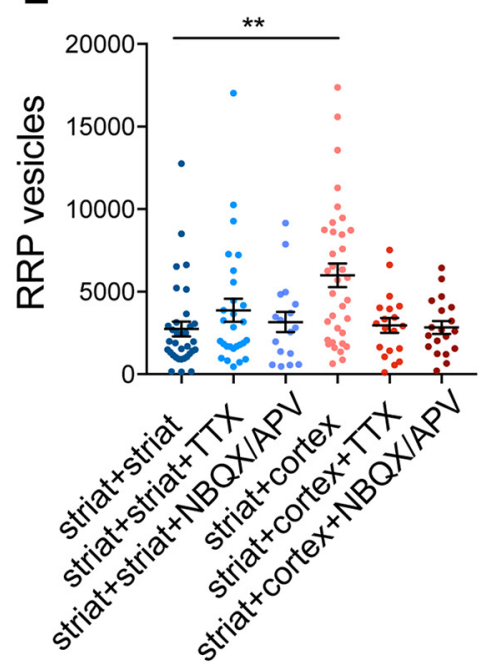

C
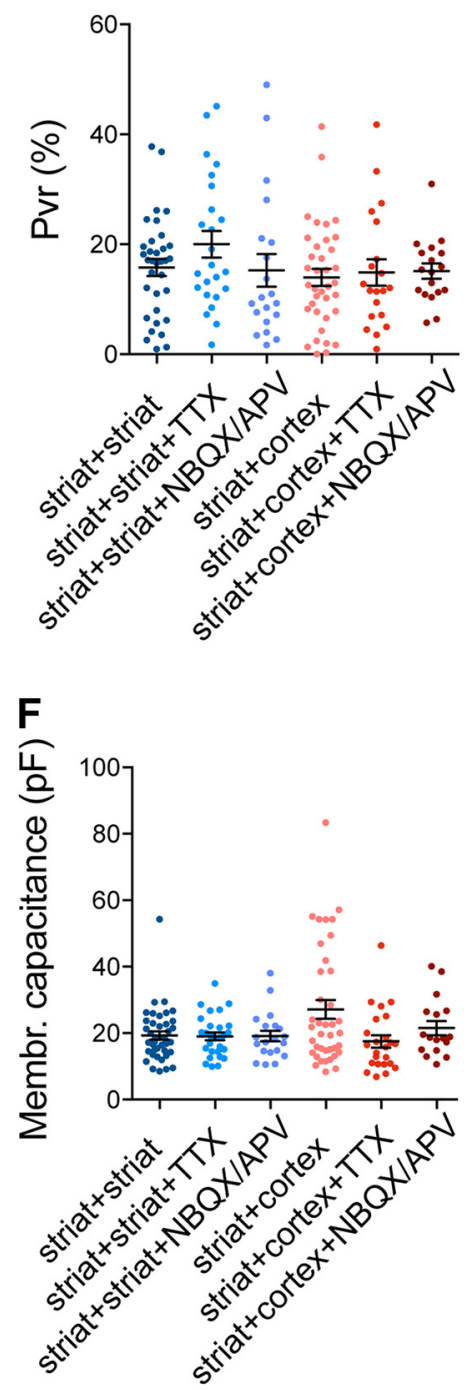

Figure 3. Activity modulates GABAergic synapse output in CS pairs. $\boldsymbol{A}-\boldsymbol{F}$, Functional analysis of striatal pairs (blue color scale dots), CS pairs (red color scale dots). Scatter plots showing total evoked IPSC amplitudes $(\boldsymbol{A})$, RRP size $(\boldsymbol{B}), \operatorname{Pvr} \%(\boldsymbol{C})$, mIPSC amplitudes $(\boldsymbol{D})$, RRP vesicle number $(\boldsymbol{E})$, and mean membrane capacitance measurements as obtained from the membrane test $(\boldsymbol{F})$. Data are mean \pm SEM. ${ }^{*} p \leq 0.05,{ }^{* *} p \leq 0.01,{ }^{* * *} p \leq 0.001,{ }^{* * * *} p \leq 0.0001$. See also Figure 3-1 (available at https://doi.org/10.1523/JNEUROSCI.2630-18.2019.f3-1).

CS pairs is largely due to an increase in the number of GABAergic synapses formed. Indeed, when we quantified the number of excitatory synapses, VGLUT1-positive puncta for CS pairs or VGLUT2-positive puncta for TS pairs, we revealed a higher number of connections formed by cortical compared with thalamic partners (CS: $312.2 \pm 38.7, n=23$; TS: $118.2 \pm 15.5, n=25, p<$ 0.0001, Mann-Whitney test) (Fig. 2C). This increased input from the cortical partners may underlie the further expansion of inhibition by the striatal neuron in this pair configuration compared with the TS pairs.

We also noted that striatal neurons tended to have an increased membrane capacitance $\left(C_{\mathrm{m}}\right)$ when cultured with either glutamatergic cells, compared with control neurons (SS: $20.22 \pm 1.32 \mathrm{pF}, n=58$; CS: $24.75 \pm 2.32 \mathrm{pF}, n=29, p=$ 0.368; TS: $24.02 \pm 2.87 \mathrm{pF}, n=24, p>0.999$, Kruskal-Wallis test) (Fig. 2D). Plausibly, changes in membrane capacitance correspond to an increase in cell surface area, and thus indicate a stimulation of growth of GABAergic neurons by glutamatergic innervation.

\section{Neuronal activity modulates GABAergic synapse formation and function in CS pairs}

To gain mechanistic insight into how glutamatergic innervation drives the morphophysiological changes in striatal GABAergic output, we went on to characterize the factors responsible for changes induced in the striatal neurons only in CS pairs. We first investigated the role of neuronal activity (Fig. 3; Fig. 3-1, available at https://doi.org/10.1523/JNEUROSCI.2630-18.2019.f3-1). To do so, we chronically inhibited neuronal activity in CS cultures with TTX $(0.5 \mu \mathrm{M})$ to block neuronal firing or glutamate receptor antagonists (2 $\mu \mathrm{M}$ NBQX, $100 \mu \mathrm{M}$ AP5) to block postsynaptic receptor activation, applied to the culture media at DIV 3, 7, 11 . We found that the IPSC amplitude in TTX-treated CS pairs was reduced by $59 \%$ and in NBQX/APV-treated CS pairs by $58 \%$, compared with untreated CS pairs (CS: $-17.93 \pm 1.87 \mathrm{nA}, n=$ 37; CS+TTX: $-7.34 \pm 1.25 \mathrm{nA}, n=21, p=0.005$; CS+NBQX/ APV: $-7.50 \pm 0.89 \mathrm{nA}, n=18, p=0.041$, Kruskal-Wallis test) (Fig. 3A). Likewise, RRP GABAergic size showed a significant decrease by $42 \%$ for TTX-treated CS pairs and by $50 \%$ for NBQX/ 
APV-treated CS pairs (CS: $-6.75 \pm 0.67 \mathrm{nC}, n=37$; CS + TTX: $-3.88 \pm 0.66 \mathrm{nC}, n=20, p=0.05$; CS+NBQX/APV: $-3.39 \pm$ $0.58 \mathrm{nC}, n=19, p=0.008$, Kruskal-Wallis test) (Fig. $3 B$ ). The release probability (Pvr) or short-term plasticity (PPR) characteristics as determined by paired pulse experiments were not significantly different after any drug application (Fig. 3C). Furthermore, we tested the effect of TTX or NBQX/APV treatment on the spontaneous release. In every case, chronic activity blockade effectively reversed the potentiation of MIPSC amplitudes upon cortical innervation back to control levels (CS: $-77.56 \pm 4.51 \mathrm{pA}, n=69$; CS+TTX: $-50.61 \pm 4.23 \mathrm{pA}, n=34$, $p=0.001$; CS+NBQX/APV: $-54.62 \pm 5.15 \mathrm{pA}, n=29, p=$ 0.028 , Kruskal-Wallis test) (Fig. $3 D$ ). The number of vesicles released onto striatal neurons in CS pairs treated with TTX or NBQX/APV tended to be decreased back to the level of control striatal pairs (SS: $2740 \pm 450.7, n=34$; CS: $5988 \pm 716.1, n=35$; CS+TTX: $2963 \pm 451.9, n=19, p=0.187$; CS+NBQX/APV: $2837 \pm 388.6, n=19, p=0.144$, Kruskal-Wallis test) (Fig. 3E) as was true also for the membrane capacitance (SS: $19.3 \pm 1.24 \mathrm{pF}$, $n=41$; CS: $27.11 \pm 2.85 \mathrm{pF}, n=39$; CS+TTX: $17.5 \pm 1.87 \mathrm{pF}$, $n=24, p=0.218$; CS +NBQX/APV: $21.53 \pm 2.12 \mathrm{pF}, n=17, p>$ 0.999, Kruskal-Wallis test) (Fig. $3 F$ ). In general, control striatal pairs treated with either TTX or NBQX/APV did not show any significant differences, compared with the untreated controls. Overall, our results indicate that, in CS pairs, the GABAergic output is significantly shaped by action potential firing and associated glutamate release from the glutamatergic neuron.

\section{BDNF elicits morphological differentiation of isolated striatal GABAergic neurons}

In addition to providing excitatory neurotransmission to a circuit, glutamatergic neurons are also the source of other important factors, including BDNF, which is crucial for GABAergic neuronal development (Park and Poo, 2013). In striatum, BDNF is considered to be released from glutamatergic synapses and not from the striatal MSNs themselves (Baydyuk and Xu, 2014). Given our findings that glutamatergic innervation stimulates formation of new synapse growth in GABAergic neurons, we investigated whether BDNF release from a cortical partner is involved in this growth.

First, we asked whether the application of BDNF alone is necessary and sufficient to induce the observed changes in striatal GABAergic output. We investigated the effects of BDNF on striatal GABAergic neuron morphology by chronically treating single striatal GABAergic neurons grown on microislands (autaptic culture) with BDNF (50 ng/ml at DIV 3, 7, 11) and performing immunocytochemical analysis at DIV 12-15 (Fig. 4). BDNF treatment resulted in a significant increase in growth of the striatal autaptic neurons. Particularly, soma size, VGAT-positive puncta, and dendritic length increased by $81 \%$ (control: $92.66 \pm$ $5.47 \mu \mathrm{m}^{2}, n=40$; BDNF: $168.1 \pm 9.21 \mu \mathrm{m}^{2}, n=50, p<0.0001$, Kruskal-Wallis test), $87 \%$ (control: $127.6 \pm 14, n=38$; BDNF: $238.5 \pm 20.03, n=46, p=0.0001$, Kruskal-Wallis test), and 75\% (control: $714.3 \pm 62.08 \mu \mathrm{m}, n=39$; BDNF: $1251 \pm 93.45 \mu \mathrm{m}$, $n=47, p<0.0001$, Kruskal-Wallis test), respectively. This growth was fully reverted by an antagonist for BDNF receptors (tyrosine receptor kinase (Trk) antagonist; K252a $200 \mathrm{nM}$ at DIV $3,7,11)\left(\mathrm{BDNF}+\mathrm{K} 252 \mathrm{a}\right.$ soma: $122.9 \pm 6.80 \mu \mathrm{m}^{2}, n=38, p=$ 0.031 ; VGAT synapses: $120.3 \pm 10.72, n=39, p=0.0002$; dendritic length: $783.7 \pm 73.33 \mu \mathrm{m} n=44, p=0.0004$, KruskalWallis test) (Fig. 4A-D; Fig. 4-1 $A$, available at https://doi.org/ 10.1523/JNEUROSCI.2630-18.2019.f4-1). In addition, we performed a series of experiments using the selective TrkB antago- nist ANA12 (10 $\mu \mathrm{M}$ at DIV 3, 7, 11). However, due to irregular toxicity effects of ANA12 on the supporting astrocytes ( $>80 \%$ of the astrocytic islands per culture were dissolved between the second and third dose at DIV 9-11; data not shown), we decided to only proceed with $\mathrm{K} 252 \mathrm{a}$. As an additional control experiment, to verify the specificity of BDNF-mediated morphological changes, we chronically treated the autaptic neurons with $50 \mathrm{ng} / \mathrm{ml}$ heatinactivated BDNF (BDNF was heat-inactivated by boiling for 5 min immediately before use at DIV 3, 7, 11). Neurons treated with heat-inactivated BDNF revealed no changes in morphology (VGAT synapses control: $116.9 \pm 8.41, n=36$; BDNF-heated: $122.9 \pm 10.8, n=39, p=0.948$, Mann-Whitney test).

We next examined the functional implications of BDNF treatment on GABAergic output of single striatal autaptic neurons (Fig. 4-1B, available at https://doi.org/10.1523/ JNEUROSCI.2630-18.2019.f4-1). Even though morphological analysis revealed a clear increase in the number of GABAergic synapses formed, BDNF-treated neurons showed a 14\% decrease in evoked IPSC amplitude (control: $-6.84 \pm 0.63 \mathrm{nA}, n=76$; BDNF: $-5.89 \pm 0.63 \mathrm{nA}, n=127, p=0.013$, Mann-Whitney test) (Fig. $4 E$ ), with no significant difference in the RRP size (control: $-3.13 \pm 0.29 \mathrm{nC}, n=76$; BDNF: $-2.47 \pm 0.17 \mathrm{nC}, n=127$, $p=0.092$, Mann-Whitney test) (Fig. $4 F$ ). As there is no apparent change in release efficiency (Fig. $4 G$ ), the slight decrease in the autaptic IPSC amplitude likely reflects a smaller quantal size. Indeed, consistent with this scenario, there was a $22 \%$ decrease in mIPSC size (control: $-50.12 \pm 2.87 \mathrm{pA}, n=74$; BDNF: $-39.27 \pm 1.88 \mathrm{pA}, n=120, p=0.0009$, Mann-Whitney test), without any change in frequency, in BDNF-treated compared with control neurons (Fig. $4 \mathrm{H}, \mathrm{J}$ ). One possible explanation for the decreased quantal size in BDNF-treated striatal autapses is that the increased number of synapses formed is not accompanied by a concomitant increase in postsynaptic GABA receptors; therefore, the same number of receptors is diluted over more synapses. To test this possibility, we compared the responses to exogenous GABA $(5 \mu \mathrm{M})$ application and found that there was no difference between BDNF-treated and control neurons (control: $1 \pm 0.1, n=8$; BDNF: $0.97 \pm 0.09, n=10, p=0.834, t$ test) (Fig. $4 K$ ), even though the BDNF-treated neurons have a significantly increased membrane capacitance (control: $17.78 \pm 1.06 \mathrm{pF}, n=$ 77; BDNF: $21.69 \pm 0.90 \mathrm{pF}, n=128, p=0.0005$, Mann-Whitney test; Fig. $4 L$ ), and thus increased membrane surface area. This suggests that while BDNF, in the absence of glutamatergic excitatory transmission, is able to induce synapse formation, it does not support an increase in functional postsynaptic GABA receptors mirroring the presynaptic changes.

\section{Activity-dependent endogenous BDNF release modulates GABAergic synapse formation and function in CS pairs}

We have shown separately that glutamatergic excitatory transmission in CS two-neuron microcircuit evokes changes in striatal GABAergic output (Fig. 3) and that exogenous application of $\mathrm{BDNF}$ to isolated striatal GABAergic neurons promotes synapse formation (Fig. 4). However, is activity-dependent BDNF release from the cortical neuron driving the changes that observed in the striatal GABAergic neurons in CS pairs? We first investigated whether, in our culture system, the source of BDNF is indeed the glutamatergic inputs and not the striatal GABAergic neurons. To this end, we compared mRNA levels using real-time PCR from pure cultures of dissociated cortex or striatum. In agreement with previous findings examining BDNF mRNA levels (Conner et al., 1997; Gharami et al., 2008), we found that the BDNF mRNA was twice as high in cortical versus striatal cultures (cortical $197 \pm$ 
$26 \%$ of striatal, $n=3, p=0.029$, MannWhitney test) (Fig. 5A), indicative of the glutamatergic inputs as a potential source of BDNF.

To test whether BDNF released from glutamatergic neurons is required for the observed changes in striatal GABAergic output, we disrupted the BDNF-TrkB receptor signaling pathway in CS and striatal only pairs (Fig. 5). We found that chronically inhibiting TrkB receptors, using the Trk inhibitor K252a, reverted the IPSC by $25 \%$ (SS: $-5.21 \pm 0.89 \mathrm{nA}, n=$ 44; CS: $-10.38 \pm 1.23 \mathrm{nA}, n=54, p=$ 0.007; CS+K252a: $-7.73 \pm 1.47 \mathrm{nA}$, $n=32, p=0.042$, Kruskal-Wallis test) and the RRP by $40 \%$ in CS pairs (SS: $-2.29 \pm 0.26 \mathrm{nC}, n=37$; CS: $-4.58 \pm$ $0.37 \mathrm{nC}, n=49, p<0.0001 ; \mathrm{CS}+\mathrm{K} 252 \mathrm{a}$ : $-2.74 \pm 0.38 \mathrm{nC}, n=31, p=0.003$, Kruskal-Wallis test) (Fig. $5 B, C$ ), back to the level of those parameters measured in striatal only pairs. Similar changes were observed for mIPSC events, where K252a inhibition caused a $30 \%$ reduction in amplitude (SS: $-42.96 \pm 2.58 \mathrm{pA}, n=40$; CS: $-66.59 \pm 4.64 \mathrm{pA}, n=63, p=0.013$; $\mathrm{CS}+\mathrm{K} 252 \mathrm{a}:-46.82 \pm 3.55 \mathrm{pA}, n=58$, $p=0.009$, Kruskal-Wallis test) (Fig. $5 E$ ). Importantly, K252a treatment in striatal only pairs induced no changes in neurotransmission (Fig. 5B-H; Fig. 5-1, available at https://doi.org/10.1523/ JNEUROSCI.2630-18.2019.f5-1), verifying the glutamatergic cortical neurons as the source of endogenous BDNF. The same findings were also confirmed by morphological analysis of pairs immunolabeled for the presynaptic marker VGAT (Fig. 5J,K). CS pairs treated with K252a antagonist revealed $46 \%$ reduction in inhibitory synapses, compared with the untreated pairs (CS: $498.5 \pm 63.2, n=32$; CS+K252a: $269.3 \pm 30.89, n=29, p=$ 0.048 , Kruskal-Wallis test).

To further investigate the specificity of endogenous BDNF release and support the involvement of the BDNF-TrkB signaling pathway in regulation of striatal synapse formation, we chronically treated CS pairs with anti-BDNF neutralizing antibody (1:100 at DIV 3, 7, 11). BDNF neutralization led to a $60 \%$ decrease in inhibitory synapses of CS pairs, compared with untreated heterotypic pairs (CS: $498.5 \pm 63.2, n=32$; CS+anti-BDNF: $197.7 \pm 22.99, n=33, p<0.0001$, Kruskal-Wallis test), thereby negating the cortical input-induced phenotype. Additionally, the number of excitatory synapses from cortical partner was reduced by $45 \%$ (CS: $570.6 \pm 52.46, n=35$; CS + anti-BDNF: $314.8 \pm 29.01, n=33$,
A

\begin{tabular}{|l|l|l|l|}
\hline Control & BDNF & K252a & BDNF + K252a \\
\hline MAP2 & & \\
\hline & & \\
\hline
\end{tabular}

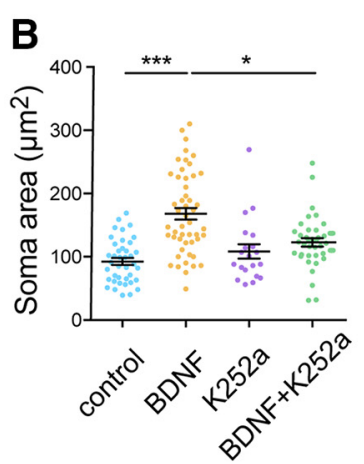

E

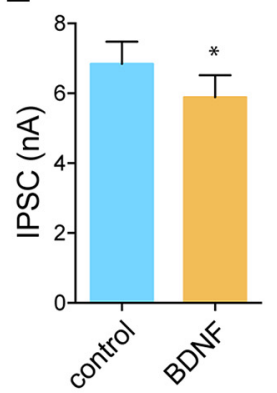

$F$

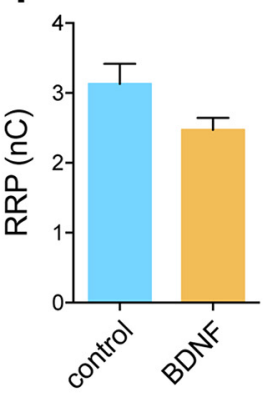

G
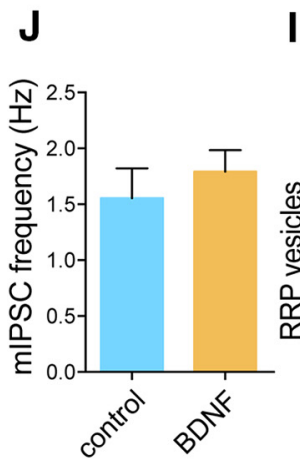

I
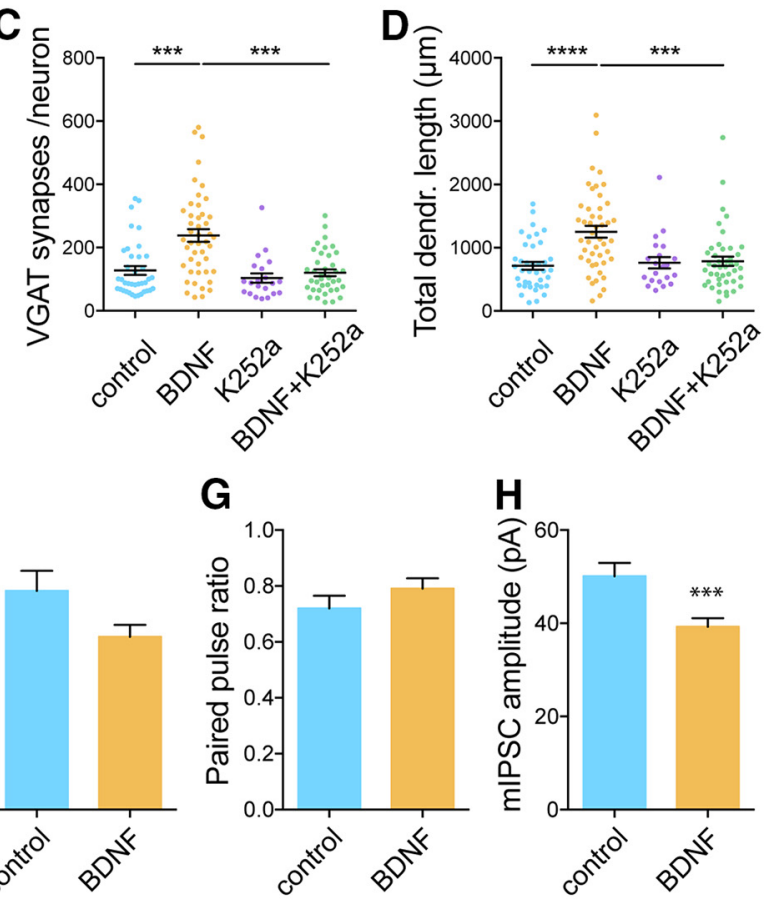

K

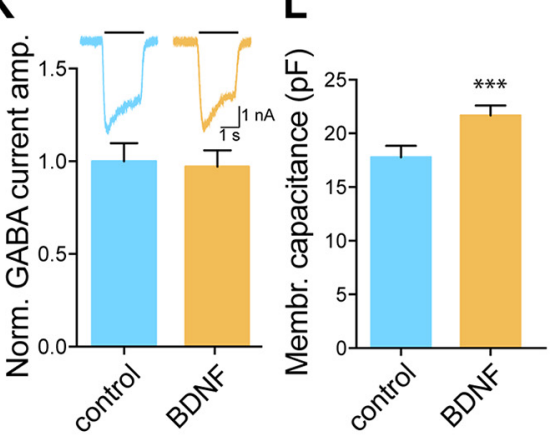

Figure 4. Exogenous BDNF promotes growth and synapse formation in striatal autaptic neurons. $\boldsymbol{A}-\boldsymbol{D}$, Morphological analysis of striatal autapses. Light blue represents control. Yellow represents BDNF-treated. Purple represents Trk-antagonist-treated. Green represents BDNF and Trk-antagonist-treated. $A$, Representative images of neuronal morphology showing immunoreactivity for MAP2 2 and VGAT. $\boldsymbol{B}-\boldsymbol{D}$, Scatter plots showing neuronal soma area $(\boldsymbol{B})$, number of VGAT synapses per neuron $(\boldsymbol{C})$, and mean total dendritic length $(\boldsymbol{D})$. $\boldsymbol{E}-\boldsymbol{L}$, Bar graphs showing evoked IPSC amplitudes $(\boldsymbol{E})$, RRP size $(\boldsymbol{F})$, PPR $(\boldsymbol{G})$, mean mIPSC amplitudes $(\boldsymbol{H})$, frequency $(\boldsymbol{J})$, RRP vesicles number $(\boldsymbol{I})$, normalized response amplitude to $5 \mu \mathrm{MGABA}(\boldsymbol{K})$, and membrane capacitance $(\boldsymbol{L})$. Data are mean \pm SEM. ${ }^{*} p \leq 0.05,{ }^{* * *} p \leq 0.001,{ }^{* * * *} p \leq 0.0001$. See also Figure $4-1$ (available at https://doi.org/10.1523/ JNEUROSCI.2630-18.2019.f4-1). 
A
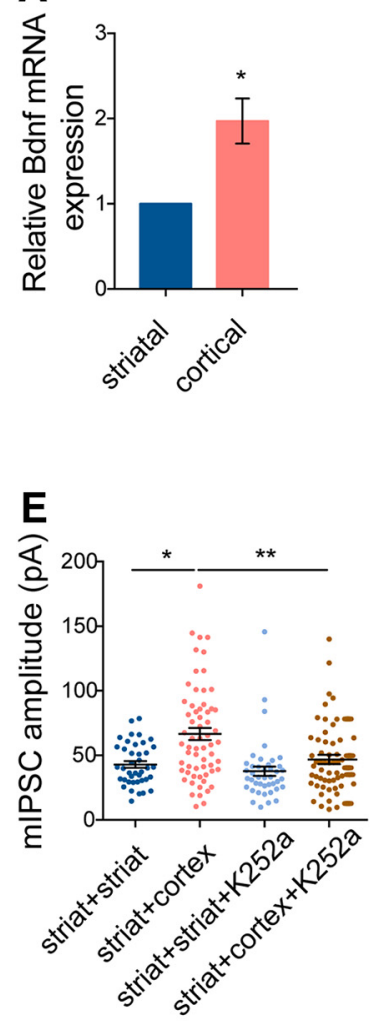

B

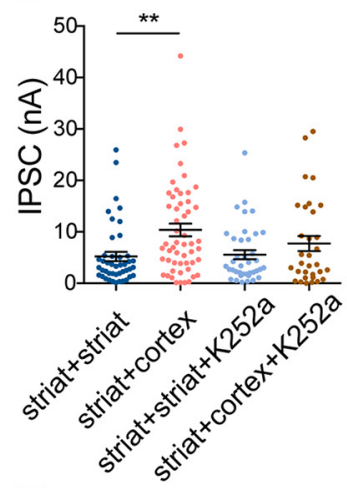

F G

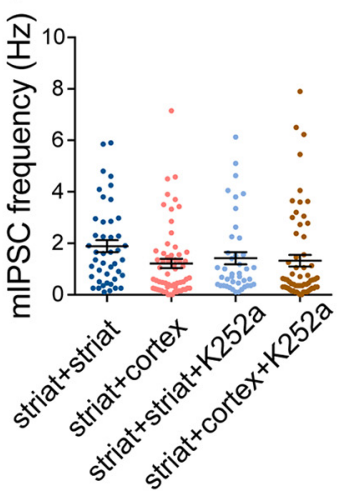

C

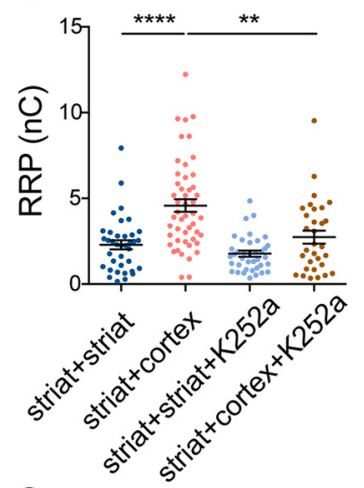

G

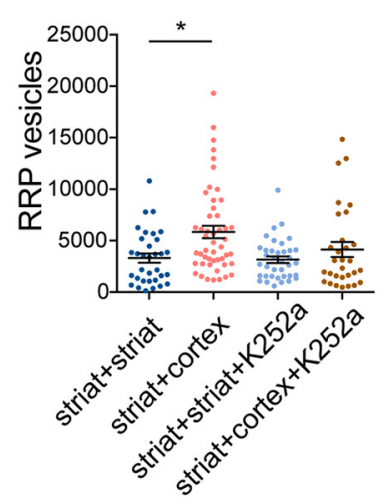

D

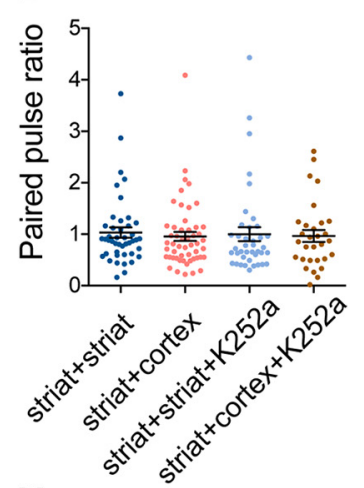

$\mathbf{H}$

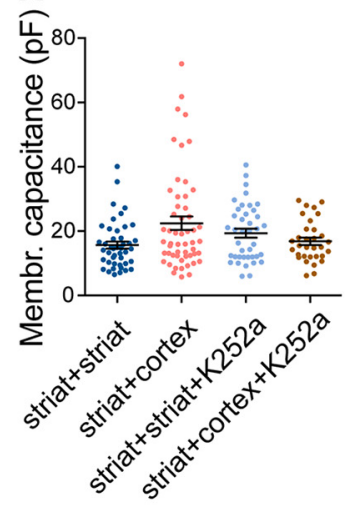

J

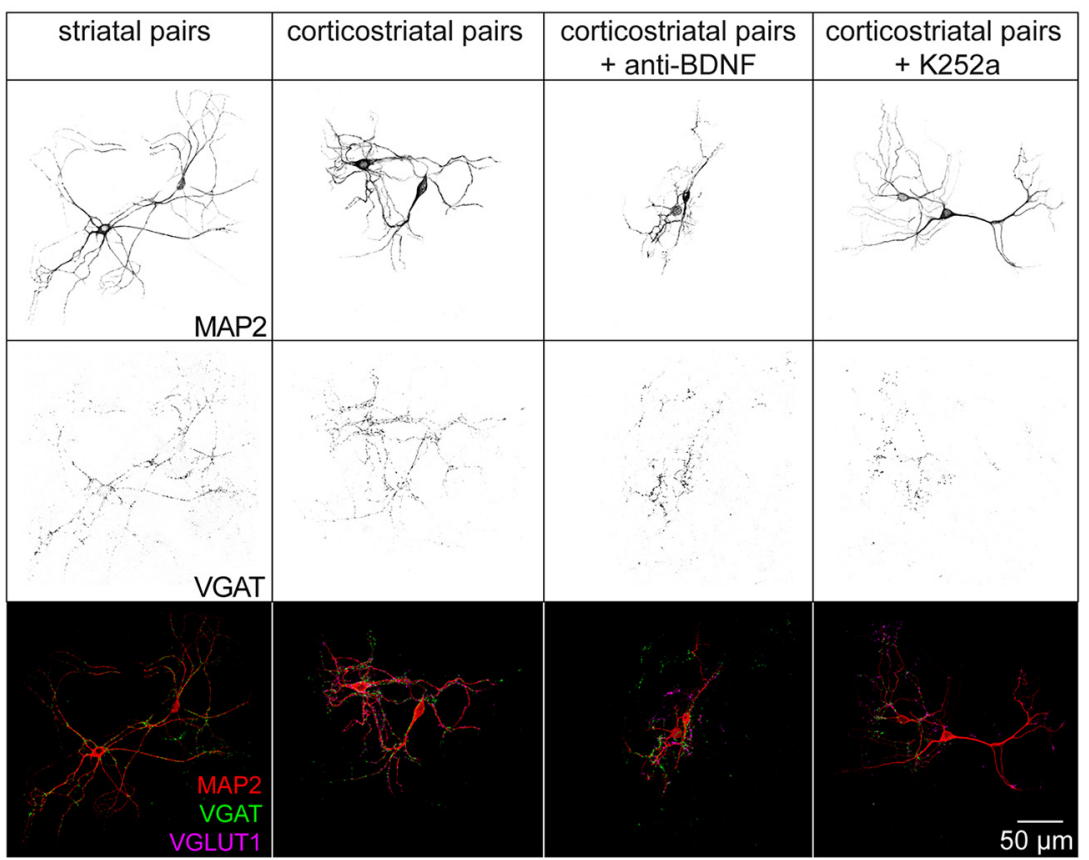

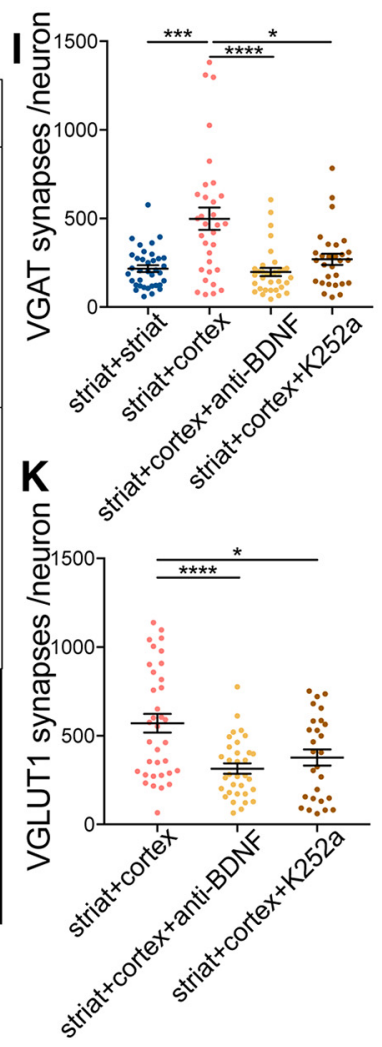

Figure 5. BDNF release modulates GABAergic synapse output in CS pairs. $A$, Bar graph showing real-time RT-PCR analysis for mRNA expression of $B d n f$ gene in striatal and cortical neuronal mass cultures. $\boldsymbol{B}-\boldsymbol{H}$, Functional analysis of striatal pairs (dark blue represents untreated; purple represents Trk-antagonist-treated), $C S$ pairs (pink represents untreated; brown represents Trk-antagonisttreated). Scatter plots showing mean evoked IPSC amplitudes (B), RRP size (C), PPR (D), mIPSC amplitudes $(\boldsymbol{E})$, mIPSC frequency $(\boldsymbol{F})$, RRP vesicle number $(\boldsymbol{G})$, and mean membrane capacitance measurements as obtained from the membrane test $(\boldsymbol{H}) . \boldsymbol{J}, \boldsymbol{K}$, Representative images of neuronal morphology showing immunoreactivity for MAP2, VGAT, and VGLUT1. Scatter plots showing the number of VGAT synapses per neuron $(\boldsymbol{I})$, the number of VGLUT1 synapses per neuron (K). Data are mean \pm SEM. ${ }^{*} p \leq 0.05,{ }^{* *} p \leq 0.01,{ }^{* * *} p \leq 0.001,{ }^{* * * *} p \leq 0.0001$. See also Figure $5-1$ (available at https://doi.org/10.1523/JNEUROSCI.2630-18.2019.f5-1). 
$p=0.001$, Kruskal-Wallis test). Together, this provides evidence that abolishing endogenous BDNF function can affect inhibitory synapse formation in striatal neurons either directly through BDNF release or by decreasing excitatory synaptic contacts and thus the level of excitation.

Overall, these data suggest that glutamatergic firing, postsynaptic glutamate receptor activation, and activity-dependent $B D N F$ release potentiate the formation of GABAergic synapses in the CS system.

\section{Discussion}

Our study investigates the potential role of glutamatergic innervation in shaping functional and morphological properties of striatal GABAergic neurons. Using a minimal circuit consisting of two neurons, we provide novel information regarding the impact of the cortical and thalamic glutamatergic input on the output synapses of the striatal GABAergic neurons. First, we report that glutamatergic input causes a twofold increase in striatal GABAergic output. With both glutamatergic partners from cortex or thalamus, we attribute this potentiation to an increase in the postsynaptic response to GABA release. Additionally, we found that cortical, but not thalamic input, enhanced the number of inhibitory synapses formed by striatal GABAergic neurons, likely as a result of the higher number of synaptic contacts that cortical neurons formed over their striatal partners. Importantly, both alterations were reverted by inhibition of neuronal activity and glutamate receptors, as well as BDNF-TrkB signaling disruption. Together, our data indicate that GABAergic synapse formation in CS pairs depends on two parallel, but potentially intersecting, signaling pathways that involve ionotropic glutamate receptor and BDNF-mediated signaling activation in striatal neurons.

\section{Cortical and thalamic glutamatergic neurons increase striatal GABAergic output}

Glutamatergic innervation from neocortex and thalamus is known to be a powerful modulator of dendritic morphology in MSNs (Segal et al., 2003; Buren et al., 2016). However, the effects of cortical and thalamic inputs on the physiology of striatal GABAergic neurons are currently understudied. Here, we denote, for the first time, that glutamatergic input from either cortex or thalamus onto striatal neurons significantly increased total GABAergic synaptic output (Fig. 1). This finding partially agrees with previous studies in the hippocampus (Chang et al., 2014; Barrows et al., 2017) that reported an increase in GABAergic output upon glutamatergic innervation, suggesting a more general role of glutamatergic input in modulating the degree of inhibition produced by GABAergic neurons. Nevertheless, the modulation by glutamatergic input in the different types of GABAergic neurons manifests in different ways: hippocampal interneurons increased the number of their output synapses, but decreased the presynaptic release efficiency (Chang et al., 2014), whereas striatal GABAergic neurons increased their evoked inhibitory response and vesicle pool size, while maintaining their release efficiency (Fig. 1G-I). A possible explanation for this discrepancy might derive from the role of each GABAergic neuronal type within its given circuitry. Interneurons are thought to function mainly locally within a circuit, and their output determines the excitation/inhibition balance (Liu, 2004; Atallah and Scanziani, 2009). On the other hand, striatal neurons project to other regions and must reliably convey information (Kreitzer and Malenka, 2008). It is thus possible that striatal neurons are optimized to be less dynamic and rather act as refiners of incoming information from other neurons. Indeed, in vivo studies of the pathways from cortex and thalamus through striatum to the downstream areas suggest that the precision of striatal neuron firing is crucial for correct representation of sensory stimulus (Smith et al., 2004; Ding et al., 2008). Our findings that striatal GABAergic neurons respond to glutamatergic input with enhanced postsynaptic response to GABA release (Fig. $1 N$ ) would support this concept. Within the striatum, direct and indirect pathway MSNs communicate with each other via local axon collaterals forming recurrent inhibitory synaptic contacts (Taverna et al., 2008). Given our finding that glutamatergic innervation causes an increase in the quantal GABA response on striatal neurons (Fig. 1), this would suggest that collateral recurrent connections within striatum could be potentiated and therefore cause more precisely timed firing in response to excitatory input. The different properties of the two classes of GABAergic neurons should come as no surprise because interneurons and projection neurons originate from different brain areas (medial and caudal vs lateral ganglionic eminence, respectively) (Angevine and Sidman, 1961; Wichterle et al., 1997; Fjodorova et al., 2015). This provides the two neuronal types with distinct transcriptional profiles and, thus, functional and morphological phenotypes.

Another interesting observation of our study is that thalamic and cortical inputs differentially affect the cellular phenotype of striatal GABAergic output. Even though both glutamatergic inputs increased postsynaptic response of striatal GABAergic neurons to GABA release (Fig. $1 N$ ), expansion of GABAergic output synapses occurred only with cortical input (Figs. $1 N, 2 B$ ). CS and TS projection systems code information in distinct ways. This could constrain the way they regulate striatal circuitry (Ding et al., 2008; Sciamanna et al., 2015) and, thus, influence its output. Given that BDNF impacts the formation of GABAergic synapses (Park and Poo, 2013), we compared $B d n f$ mRNA levels between cortical and thalamic mass cultures. Yet, we found no differences (data not shown). However, in morphological analysis of our pairs, the number of cortical synapses made in CS pairs was higher than those in TS pairs (Fig. 2C). Therefore, we presume that the explanation for the divergent effects of cortical versus thalamic input on striatal output stems from the overall strength of the synaptic input provided by each of the glutamatergic partners. Indeed, in agreement with other studies (Smeal et al., 2007; Kolodziejczyk and Raymond, 2016), we found that, in addition to showing morphologically fewer synapses, thalamic neuronevoked responses were smaller compared with those evoked by cortical neurons (Fig. $1 R, S$ ). This suggests that only strong synaptic input driven by cortical neurons might be able to induce the formation of additional inhibitory synapses in striatal GABAergic neurons.

\section{Cortical-induced potentiation of striatal GABAergic output requires neuronal activity and activity-dependent BDNF release}

The mechanisms through which striatal GABAergic neurons respond to glutamatergic innervation are currently unclear. We know from previous studies that excitation and activation of ionotropic glutamate receptors regulate GABAergic synapse formation in interneurons (Hartman et al., 2006; Chang et al., 2014). Furthermore, Segal et al. (2003) showed that, in CS embryonic cultures, the presence of TTX in growth media prevented the increase in the density of striatal spines caused by cortical input. In this sense, our study verifies the importance of neuronal firing and glutamate receptor activation for GABAergic neuronal function, showing now that glutamatergic excitation not only 
alters striatal spines density, but it is also relevant for modulating GABAergic output (Fig. 3).

Another potential component of cortical glutamatergic innervation that impacts GABAergic output is activity-dependent BDNF release (Huang et al., 1999; Hong et al., 2008). In this context, we showed that the source of BDNF more likely comes from glutamatergic fibers, as $B d n f$ mRNA expression was substantially higher in cortical than striatal mass culture (Fig. 5A). Furthermore, we demonstrated, for the first time, that application of Trk receptor antagonist, K252a, prevented the increase in GABAergic output caused by cortical input, suggesting that BDNF is required for both quantal response changes and inhibitory synapse formation (Fig. 5). This was also supported by the experiments using anti-BDNF neutralizing antibody (Fig. $5 \mathrm{~J}, \mathrm{~K}$ ). This extends previous findings on the role of BDNF as a regulator of cellular and dendritic morphology for striatal GABAergic neurons (Nakao et al., 1995; Palizvan et al., 2004; Rauskolb et al., 2010; Penrod et al., 2015). Nevertheless, despite the fact that BDNF directly influences the morphology (cell size, dendritic length, and number of contacts), we showed that BDNF alone is not sufficient to induce new, fully functional synapses in striatal neurons (Fig. 4). We presume that activity-dependent BDNF release by cortical glutamatergic neurons activates TrkB receptors in striatal cells and turns on the transcriptional programs instructing synapse formation (Hong et al., 2008). As a result, in conjunction with neuronal activity, cortical input regulates the maintenance of those synapses (Marty et al., 2000; Lin et al., 2008; West and Greenberg, 2011). To shed further light on this mechanism, future studies examining gene expression changes are required.

In conclusion, our study demonstrates the power of an in vitro approach to examine interregional interactions between different neuronal types that are known to form specific circuits in vivo. Using this two-neuron in vitro system, we were able to isolate the specific role of glutamatergic innervation onto striatal GABAergic output, examine specific parameters underlying synaptic function and study putative factors required for glutamatergic-induced modulation. Nevertheless, to strengthen the relevance of our findings, future studies examining striatal cell modulation in vivo are necessary. Nowadays, that cumulative evidence support that basal ganglia circuit deficits are implicated in numerous neurological diseases, including Huntington's disease (Cepeda et al., 2003) and Tourette and Parkinson's syndromes, we are confident that our two-neuron microcircuit model could be a valuable tool for assessing synaptic properties of such disease models in cellular context. Understanding how cortical and thalamic inputs refine striatal output will pave the way toward identifying basal ganglia activity in both physiological and pathological conditions.

\section{References}

Albin RL, Young AB, Penney JB (1989) The functional anatomy of basal ganglia disorder. Trends Neurosci 12:366-375.

Angevine JB Jr, Sidman RL (1961) Autoradiographic study of cell migration during histogenesis of cerebral cortex in the mouse. Nature 192:766-768.

Atallah BV, Scanziani M (2009) Instantaneous modulation of gamma oscillation frequency by balancing excitation with inhibition. Neuron 62:566577.

Barrows CM, McCabe MP, Chen H, Swann JW, Weston MC (2017) PTEN loss increases the connectivity of fast synaptic motifs and functional connectivity in a developing hippocampal network. J Neurosci 37:85958611.

Baydyuk M, Xu B (2014) BDNF signaling and survival of striatal neurons. Front Cell Neurosci 8:254.

Buren C, Tu G, Parsons MP, Sepers MD, Raymond LA (2016) Influence of cortical synaptic input on striatal neuronal dendritic arborization and sensitivity to excitotoxicity in corticostriatal co-culture. J Neurophysiol 116:380-390.

Cepeda C, Hurst RS, Calvert CR, Hernández-Echeagaray E, Nguyen OK, Jocoy E, Christian LJ, Ariano MA, Levine MS (2003) Transient and progressive electrophysiological alterations in the corticostriatal pathway in a mouse model of Huntington's disease. J Neurosci 23:961-969.

Chang CL, Trimbuch T, Chao HT, Jordan JC, Herman MA, Rosenmund C (2014) Investigation of synapse formation and function in a glutamatergic-GABAergic two-neuron microcircuit. J Neurosci 34:855-868.

Cohen J (1988) Statistical power analysis for the behavioral sciences. New York, NY: Lawrence Erlbaum Associates.

Conner JM, Lauterborn JC, Yan Q, Gall CM, Varon S (1997) Distribution of brain-derived neurotrophic factor (BDNF) protein and mRNA in the normal adult rat CNS: evidence for anterograde axonal transport. J Neurosci 17:2295-2313.

Cui G, Jun SB, Jin X, Pham MD, Vogel SS, Lovinger DM, Costa RM (2013) Concurrent activation of striatal direct and indirect pathways during action initiation. Nature 494:238-242.

Czubayko U, Plenz D (2002) Fast synaptic transmission between striatal spiny projection neurons. Proc Natl Acad Sci U S A 99:15764-15769.

del Castillo BY, Katz B (1954) Quantal components of the end-plate potential. J Physiol 124:560-573.

DeLong MR (1990) Primate models of movement disorders of basal ganglia origin. Trends Neurosci 13:281-285.

Ding J, Peterson JD, Surmeier DJ (2008) Corticostriatal and thalamostriatal synapses have distinctive properties. J Neurosci 28:6483-6492.

Doig NM, Moss J, Bolam JP (2010) Cortical and thalamic innervation of direct and indirect pathway medium-sized spiny neurons in mouse striatum. J Neurosci 30:14610-14618.

Fjodorova M, Noakes Z, Li M (2015) How to make striatal projection neurons. Neurogenesis 2:1-6.

Fremeau RT Jr, Troyer MD, Pahner I, Nygaard GO, Tran CH, Reimer RJ, Bellocchio EE, Fortin D, Storm-Mathisen J, Edwards RH (2001) The expression of vesicular glutamate transporters defines two classes of excitatory synapse. Neuron 31:247-260.

Fujiyama F, Furuta T, Kaneko T (2001) Immunocytochemical localization of candidates for vesicular glutamate transporters in the rat cerebral cortex. J Comp Neurol 435:379-387.

Gerfen CR (1992) The neostriatal mosaic: multiple levels of compartmental organization. Trends Neurosci 15:133-139.

Gharami K, Xie Y, An JJ, Tonegawa S, Xu B (2008) Brain-derived neurotrophic factor over-expression in the forebrain ameliorates Huntington's disease phenotypes in mice. J Neurochem 105:369-379.

Groenewegen HJ, Berendse HW (1994) The specificity of the "nonspecific" midline and intralaminar thalamic nuclei. Trends Neurosci 17:52-57.

Hartman KN, Pal SK, Burrone J, Murthy VN (2006) Activity-dependent regulation of inhibitory synaptic transmission in hippocampal neurons. Nat Neurosci 9:642-649.

Hong EJ, McCord AE, Greenberg ME (2008) A biological function for the neuronal activity-dependent component of BDNF transcription in the development of cortical inhibition. Neuron 60:610-624.

Huang ZJ, Kirkwood A, Pizzorusso T, Porciatti V, Morales B, Bear MF, Maffei L, Tonegawa S (1999) BDNF regulates the maturation of inhibition and the critical period of plasticity in mouse visual cortex. Cell 98:739-755.

Kemp JM, Powell TP (1970) The cortico-striate projection in the monkey. Brain 93:525-546.

Kolodziejczyk K, Raymond LA (2016) Differential changes in thalamic and cortical excitatory synapses onto striatal spiny projection neurons in a Huntington disease mouse model. Neurobiol Dis 86:62-74.

Kreitzer AC, Malenka RC (2008) Striatal plasticity and basal ganglia circuit function. Neuron 60:543-554.

Lin Y, Bloodgood BL, Hauser JL, Lapan AD, Koon AC, Kim TK, Hu LS, Malik AN, Greenberg ME (2008) Activity-dependent regulation of inhibitory synapse development by Npas4. Nature 455:1198-1204.

Liu G (2004) Local structural balance and functional interaction of excitatory and inhibitory synapses in hippocampal dendrites. Nat Neurosci 7:373-379.

Liu H, Dean C, Arthur CP, Dong M, Chapman ER (2009) Autapses and networks of hippocampal neurons exhibit distinct synaptic transmission phenotypes in the absence of synaptotagmin I. J Neurosci 29:7395-7403.

Liu H, Chapman ER, Dean C (2013) “Self" versus “non-self” connectivity 
dictates properties of synaptic transmission and plasticity. PLoS One 8:1-9.

Marty S, Wehrlé R, Sotelo C (2000) Neuronal activity and brain-derived neurotrophic factor regulate the density of inhibitory synapses in organotypic slice cultures of postnatal hippocampus. J Neurosci 20:8087-8095.

McGeorge AJ, Faull RL (1989) The organization of the projection from the cerebral cortex to the striatum in the rat. Neuroscience 29:503-537.

Meijering E, Jacob M, Sarria JC, Steiner P, Hirling H, Unser M (2004) Design and validation of a tool for neurite tracing and analysis in fluorescence microscopy images. Cytometry 58:167-176.

Nakao N, Brundin P, Funa K, Lindvall O, Odin P (1995) Trophic and protective actions of brain-derived neurotrophic factor on striatal DARPP32-containing neurons in vitro. Dev Brain Res 90:92-101.

Oldenburg IA, Sabatini BL (2015) Antagonistic but not symmetric regulation of primary motor cortex by basal ganglia direct and indirect pathways. Neuron 86:1174-1181.

Palizvan MR, Sohya K, Kohara K, Maruyama A, Yasuda H, Kimura F, Tsumoto T (2004) Brain-derived neurotrophic factor increases inhibitory synapses, revealed in solitary neurons cultured from rat visual cortex. Neuroscience 126:955-966.

Park H, Poo MM (2013) Neurotrophin regulation of neural circuit development and function. Nat Rev Neurosci 14:7-23.

Penrod RD, Campagna J, Panneck T, Preese L, Lanier LM (2015) The presence of cortical neurons in striatal-cortical co-cultures alters the effects of dopamine and BDNF on medium spiny neuron dendritic development. Front Cell Neurosci 9:1-14.

Randall FE, Garcia-Munoz M, Vickers C, Schock SC, Staines WA, Arbuthnott GW (2011) The corticostriatal system in dissociated cell culture. Front Syst Neurosci 5:1-7.

Rauskolb S, Zagrebelsky M, Dreznjak A, Deogracias R, Matsumoto T, Wiese S, Erne B, Sendtner M, Schaeren-Wiemers N, Korte M, Barde YA (2010) Global deprivation of brain-derived neurotrophic factor in the CNS reveals an area-specific requirement for dendritic growth. J Neurosci 30:1739-1749.

Reim K, Mansour M, Varoqueaux F, McMahon HT, Südhof TC, Brose N, Rosenmund C (2001) Complexins regulate a late step in $\mathrm{Ca}^{2+}$ dependent neurotransmitter release. Cell 104:71-81.

Rosenmund C, Stevens CF, Zellula AG (1996) Definition of the readily releasable pool of vesicles at hippocampal synapses. Neuron 16:1197-1207.

Rothwell PE, Hayton SJ, Sun GL, Fuccillo MV, Hopkins K, Kook Lim BK,
Malenka RC (2015) Input- and output-specific regulation of serial order performance by corticostriatal circuits. Neuron 88:345-356.

Salin P, Kachidian P (1998) Thalamo-striatal deafferentation affects preproenkephalin but not preprotachykinin gene expression in the rat striatum. Mol Brain Res 57:257-265.

Sciamanna G, Ponterio G, Mandolesi G, Bonsi P, Pisani A (2015) Optogenetic stimulation reveals distinct modulatory properties of thalamostriatal vs corticostriatal glutamatergic inputs to fast-spiking interneurons. Sci Rep 5:16742.

Segal M, Greenberger V, Korkotian E (2003) Formation of dendritic spines in cultured striatal neurons depends on excitatory afferent activity. Eur J Neurosci 17:2573-2585.

Smeal RM, Gaspar RC, Keefe KA, Wilcox KS (2007) A rat brain slice preparation for characterizing both thalamostriatal and corticostriatal afferents. J Neurosci Methods 159:224-235.

Smith AD, Bolam JP (1990) The neural network of the basal ganglia as revealed by the study of synaptic connections of identical neurones. Trends Neurosci 13:259-265.

Smith Y, Raju DV, Pare JF, Sidibe M (2004) The thalamostriatal system: a highly specific network of the basal ganglia circuitry. Trends Neurosci 27:520-527.

Stern EA, Kincaid AE, Wilson CJ (1997) Spontaneous subthreshold membrane potential fluctuations and action potential variability of rat corticostriatal and striatal neurons in vivo. J Neurophysiol 77:1697-1715.

Taverna S, Ilijic E, Surmeier DJ (2008) Recurrent collateral connections of striatal medium spiny neurons are disrupted in models of Parkinson's disease. J Neurosci 28:5504-5512.

Tunstall MJ, Oorschot DE, Kean A, Wickens JR (2002) Inhibitory interactions between spiny projection neurons in the rat striatum. J Neurophysiol 88:1263-1269.

West AE, Greenberg ME (2011) Neuronal activity-regulated gene transcription in synapse development and cognitive function. Cold Spring Harb Perspect Biol 3:1-21.

Wichterle H, Garcia-Verdugo JM, Alvarez-Buylla A (1997) Direct evidence for homotypic, glia-independent neuronal migration. Neuron 18:779791.

Wilson CJ (1993) The generation of natural firing patterns in neostiatal neurons. Prog Brain Res 99:277-297.

Wilson CJ (2014) The sensory striatum. Neuron 83:999-1001. 